\title{
Porosity and differentiability of Lipschitz maps from stratified groups to Banach homogeneous groups
}

\author{
Valentino Magnani ${ }^{1}$ (D) Andrea Pinamonti ${ }^{2} \cdot$ Gareth Speight $^{3}$ \\ Received: 30 October 2018 / Accepted: 27 September 2019 / Published online: 9 October 2019 \\ (c) Fondazione Annali di Matematica Pura ed Applicata and Springer-Verlag GmbH Germany, part of Springer \\ Nature 2019
}

\begin{abstract}
Let $f$ be a Lipschitz map from a subset $A$ of a stratified group to a Banach homogeneous group. We show that directional derivatives of $f$ act as homogeneous homomorphisms at density points of $A$ outside a $\sigma$-porous set. At all density points of $A$, we establish a pointwise characterization of differentiability in terms of directional derivatives. These results naturally lead us to an alternate proof of almost everywhere differentiability of Lipschitz maps from subsets of stratified groups to Banach homogeneous groups satisfying a suitably weakened Radon-Nikodym property.
\end{abstract}

Keywords Stratified group · Carnot group - Banach homogeneous group · Carnot-Carathéodory distance $\cdot$ Lipschitz map · Differentiability $\cdot$ Porous set

Mathematics Subject Classification 28A75 · 43A80 - 49Q15 · 53C17

\section{Introduction}

Stratified groups are a special class of finite dimensional, connected, simply connected and nilpotent Lie groups (Definition 2.4). These groups, equipped with the so-called homoge-

V. M. acknowledges the support of the University of Pisa, (Institutional Research Grant) Project PRA 2016 41 and Project PRA 2018 49. A. P. acknowledges the support of the Istituto Nazionale di Alta Matematica F. Severi.

\footnotetext{
$凶$ Valentino Magnani

valentino.magnani@unipi.it

Andrea Pinamonti

andrea.pinamonti@unitn.it

Gareth Speight

Gareth.Speight@uc.edu

1 Department of Mathematics, University of Pisa, Largo Pontecorvo 5, 56127 Pisa, Italy

2 Department of Mathematics, University of Trento, Via Sommarive 14, 38123 Povo (Trento), Italy

3 Department of Mathematical Sciences, University of Cincinnati, 2815 Commons Way, Cincinnati, OH 45221, USA
} 
neous norm, were introduced by Folland in the framework of subelliptic PDE [14]. They subsequently appeared in the work of Pansu under the name of Carnot groups, where their metric structure was defined by the so-called Carnot-Carathéodory distance [29]. In the last two decades, there has been increasing interest in the relationship between the geometry of stratified groups and other areas of mathematics, such as PDE, differential geometry, control theory, geometric measure theory, mathematical finance and robotics $[1,5,6,11,17,28,38]$.

Nevertheless, several interesting and challenging problems remain open, since the geometry of stratified groups is highly non-trivial. In this respect, we mention rectifiability of the reduced boundary in higher step groups, the isoperimetric problem, the regularity of minimal surfaces, the regularity of geodesics and the validity of general coarea formulae for Lipschitz mappings. One of the basic reasons for these difficulties is that any noncommutative stratified group contains no subset of positive measure which is bi-Lipschitz equivalent to a subset of a Euclidean space [3,18,25,37]. In all of these results, a key role is played by Pansu's generalization of Rademacher's theorem to Carnot groups [29].

This differentiation theorem states that every Lipschitz map from an open subset of a stratified group to another stratified group is differentiable almost everywhere with respect to the natural Haar measure. Here, differentiability is defined like classical differentiability, but takes into account the new geometric and algebraic structure (Definition 2.18). More broadly, a great effort has been made to understand differentiability properties of Lipschitz functions in different settings, like stratified groups, Banach spaces and more general metric measure spaces. We mention only a few papers $[2,4,7-10,16,21,22,26,30,34-36]$ to give a glimpse of the many works in this area.

In the setting of noncommutative stratified groups, the first named author and Rajala extended Pansu's theorem to Lipschitz mappings on a measurable set of a stratified group and taking values in a Banach homogeneous group (Definition 2.3). They proved the following theorem [27].

Theorem 1.1 Suppose $\mathbb{M}$ is a Banach homogeneous group whose first layer $H_{1} \subset \mathbb{M}$ has the RNP. If $f: A \rightarrow \mathbb{M}$ is Lipschitz, $A \subset \mathbb{G}$ and $\mathbb{G}$ is a stratified group, then $f$ is almost everywhere differentiable.

It is easy to notice that every stratified group is a Banach homogeneous group, and the class of finite-dimensional Banach homogeneous groups coincides with that of homogeneous groups, as defined in [13]. Commutative Banach homogeneous groups coincide with Banach spaces. The simplest example besides these is an infinite-dimensional version of the Heisenberg group $H^{2} \times \mathbb{R}$. We consider the infinite-dimensional real Hilbert space $H$ with scalar product $\langle\cdot, \cdot\rangle$, and the group operation is defined as follows:

$$
\left(h_{1}, h_{2}, t_{1}\right) \cdot\left(h_{1}^{\prime}, h_{2}^{\prime}, t_{1}^{\prime}\right)=\left(h_{1}+h_{1}^{\prime}, h_{2}+h_{2}^{\prime}, t_{1}+t_{2}+\left\langle h_{1}, h_{2}^{\prime}\right\rangle-\left\langle h_{2}, h_{1}^{\prime}\right\rangle\right),
$$

for any $\left(h_{1}, h_{2}, t_{1}\right),\left(h_{1}^{\prime}, h_{2}^{\prime}, t_{1}^{\prime}\right) \in H^{2} \times \mathbb{R}$. We refer the interested reader to [27] for more examples.

The present paper continues the investigation started in [31], on the relationship between porosity and differentiability of Lipschitz maps on stratified groups. A set in a metric space is (upper) porous (Definition 2.19) if each of its points sees nearby relatively large holes in the set on arbitrarily small scales. A set is $\sigma$-porous if it is a countable union of porous sets. Proving a set is $\sigma$-porous is useful because $\sigma$-porous sets in metric spaces are first category and have measure zero with respect to any doubling measure $[39,40]$. Showing a set is $\sigma$-porous usually gives a stronger result than showing it is of first category or has measure zero. 
It is well known that porosity is a useful tool to study differentiability of Lipschitz mappings. We refer the reader to the survey articles [39,40] for more information about porous sets and their applications. Porosity has been also recently used in Euclidean spaces to obtain the following result: for any $n>1$, there exists a Lebesgue null set in $\mathbb{R}^{n}$ containing a point of differentiability for every Lipschitz mapping from $\mathbb{R}^{n}$ to $\mathbb{R}^{n-1}$ [35]. In the Banach space setting, [22,23] give a version of Rademacher's theorem for Frechét differentiability of Lipschitz mappings on Banach spaces in which porous sets are negligible in a suitable sense. Other results have also been studied in stratified groups [20,30,32,33].

In [31], the second and third named authors used porosity to study some fine differentiability properties of Lipschitz mappings from stratified groups to Euclidean spaces. In the present paper, we improve upon [31] in two directions: we consider more general classes of domains and targets of the Lipschitz mapping. We allow the mapping to be defined on a measurable subset of a stratified group. Due to the absence in general of Lipschitz extensions between stratified groups, some technical difficulties appear. The Euclidean space in the target is replaced by a Banach homogeneous group. Thus, the noncommutative infinite-dimensional structure of a Banach homogeneous group adds further difficulties.

Let $A$ be a subset of a stratified group $\mathbb{G}$ and $\mathbb{M}$ be a Banach homogeneous group, and consider a Lipschitz map $f: A \subset \mathbb{G} \rightarrow \mathbb{M}$. Our first result states that directional derivatives of $f$ at density points of the domain act as homogeneous homomorphisms outside a $\sigma$-porous set. Here, it is worth to emphasize that the notion of directional derivative (Definition 2.9) takes into account the fact that $f$ need not be defined on a whole neighborhood of the point. For the definition of homogeneous homomorphism, see Definition 2.17. Note that $\partial^{+} f(x, \zeta)$ and $\delta_{a} \zeta$ denote directional derivatives and dilations, as defined in Sect. 2 and $D(A)$ denotes the set of density points of $A \subset \mathbb{G}$.

Theorem 1.2 Suppose $A \subset \mathbb{G}$ is measurable and $f: A \rightarrow \mathbb{M}$ is Lipschitz. Then, there is a $\sigma$-porous set $P \subset \mathbb{G}$ such that whenever $x \in A \cap D(A) \backslash P$, the following implication holds: if $\partial^{+} f(x, \zeta)$ and $\partial^{+} f(x, \eta)$ exist for some $\zeta, \eta \in \mathbb{G}$, then $\partial^{+} f\left(x, \delta_{a} \zeta \delta_{b} \eta\right)$ exists for every $a, b>0$ and

$$
\partial^{+} f\left(x, \delta_{a} \zeta \delta_{b} \eta\right)=\delta_{a} \partial^{+} f(x, \zeta) \delta_{b} \partial^{+} f(x, \eta)
$$

Surprisingly, the techniques of [36, Theorem 2] can be extended to the nonlinear framework of stratified groups. Nontrivial obstacles arise from the structure of both the domain and the groups involved. Theorem 1.2 precisely generalizes [31, Theorem 3.7] from real-valued Lipschitz mappings to Banach homogeneous group-valued Lipschitz mappings.

For real-valued Lipschitz mappings, directional derivatives were defined using the Lie algebra of the stratified group and only horizontal directions were used. In the present paper, we realize that directional derivatives must be introduced for all directions, using the Lie group structures of $\mathbb{G}$ and $\mathbb{M}$. This important difference is necessary to reflect the noncommutative structure of the target, and it was rather unexpected. We notice, indeed, that the restriction of a Lipschitz mapping to a nonhorizontal curve is no longer Lipschitz in the Euclidean sense.

Our second main result characterizes points of differentiability at density points of the domain. For the definition of directional derivatives, see Definition 2.9.

Theorem 1.3 Suppose $A \subset \mathbb{G}$ is measurable, $f: A \rightarrow \mathbb{M}$ is Lipschitz and $x \in A \cap D(A)$. Then, $f$ is differentiable at $x$ if and only if the following properties hold:

(1) $f$ is differentiable at $x$ in any direction $\zeta \in \mathbb{G} \backslash\{0\}$

(2) the mapping $\mathbb{G} \ni \zeta \rightarrow \partial^{+} f(x, \zeta) \in \mathbb{M}$ is a homogeneous homomorphism. 
As a consequence of Theorems 1.2 and 1.3, we prove that, at density points of the domain except for a $\sigma$-porous set, existence of directional derivatives in a spanning set of horizontal directions implies differentiability. If the horizontal space of $\mathbb{M}$ satisfies the RNP, then directional derivatives in this finite set of directions exist at almost every point of $A$ (Theorem 2.16). As a by-product, taking into account that porous sets have measure zero, we are lead to a different and simpler way to establish both Theorem 1.1 and then also Pansu's Theorem [29].

The results of this paper illustrate the role played by porosity to pass from almost everywhere existence of horizontal directional derivatives [27, Theorem 3.1] to almost everywhere differentiability of Lipschitz maps. The use of porosity provides a more natural approach to differentiability and provides more precise information about how differentiability occurs.

We now describe the structure of the paper. In Sect. 2, we give the main definitions. In Sect. 3, we prove Theorem 1.2, while in Sect. 4, we prove Theorem 1.3. Section 5 is devoted to a few technical facts. The first one is the construction of a separable Banach homogeneous target for our given Lipschitz mapping. We then give a proof of a technical lemma that we use earlier for estimating distances. Finally, we prove that the set of points where $f$ is differentiable in some directions is measurable. This measurability is important to establish Theorem 2.16, and its proof is rather technical since our Lipschitz mapping cannot be extended to the whole space.

\section{Preliminaries}

This section describes the main notions used throughout the paper.

\subsection{Banach Lie algebras and Banach homogeneous groups}

We recall only basic facts about Banach homogeneous groups. More information and additional examples can be found in [27].

Definition 2.1 A Banach Lie algebra is a Banach space $\mathbb{M}$ equipped with a continuous Lie bracket, namely a continuous, bilinear and skew-symmetric mapping $[\cdot, \cdot]: \mathbb{M} \times \mathbb{M} \longrightarrow \mathbb{M}$ that satisfies the Jacobi identity:

$$
[x,[y, z]]+[y,[z, x]]+[z,[x, y]]=0 \quad \text { for all } x, y, z \in \mathbb{M} .
$$

A Banach Lie algebra $\mathbb{M}$ is nilpotent if there is $v \in \mathbb{N}$ such that whenever $x_{1}, x_{2}, \ldots, x_{v+1} \in$ $\mathbb{M}$, we have

$$
\left[\left[\left[\cdots\left[\left[x_{1}, x_{2}\right], x_{3}\right] \cdots\right], x_{v}\right], x_{v+1}\right]=0,
$$

and there exist $y_{1}, y_{2}, \ldots, y_{v} \in \mathbb{M}$ such that

$$
\left[\left[\cdots\left[\left[y_{1}, y_{2}\right], y_{3}\right] \cdots\right], y_{\nu}\right] \neq 0 \text {. }
$$

The integer $v$ is uniquely defined and is called the step of nilpotence of $\mathbb{M}$.

A nilpotent Banach Lie algebra $\mathbb{M}$ can be equipped with the Lie group operation:

$$
x y=x+y+\sum_{m=2}^{v} P_{m}(x, y),
$$


which is the truncated Baker-Campbell-Hausdorff series where the Banach Lie bracket is used. For any $m \geq 2$, the polynomial $P_{m}$ above is given by Dynkin's formula:

$$
P_{m}(x, y)=\sum \frac{(-1)^{k-1}}{k} \frac{m^{-1}}{p_{1} ! q_{1} ! \cdots p_{k} ! q_{k} !} \underbrace{x \circ \cdots \circ x}_{p_{1} \text { times }} \circ \overbrace{y \circ \cdots \circ y}^{q_{1} \text { times }} \cdots \circ \underbrace{x \circ \cdots \circ x}_{p_{k} \text { times }} \circ \overbrace{y \circ \cdots \circ y}^{q_{k} \text { times }} .
$$

Here, we used the nonassociative product:

$$
x_{i_{1}} \circ x_{i_{2}} \circ \cdots \circ x_{i_{k}}=\left[\left[\cdots\left[\left[x_{i_{1}}, x_{i_{2}}\right], x_{i_{3}}\right] \cdots\right], x_{i_{k}}\right],
$$

and the sum is taken over the $2 k$-tuples $\left(p_{1}, q_{1}, p_{2}, q_{2}, \ldots, p_{k}, q_{k}\right)$ such that $p_{i}+q_{i} \geq 1$ for all positive $i, k \in \mathbb{N}$ and $\sum_{i=1}^{k} p_{i}+q_{i}=m$. Note that $P_{2}$ has the simple form $P_{2}(x, y)=$ $[x, y] / 2$. The explicit formula for the group product will only be used in the technical Section 5 .

Definition 2.2 A nilpotent Banach Lie algebra equipped with the group operation (2.1) is called a Banach nilpotent Lie group.

If $S_{1}, S_{2}, \ldots, S_{n} \subset \mathbb{X}$ are closed subspaces of a Banach space $\mathbb{X}$ such that the mapping

$$
J: S_{1} \times \cdots \times S_{n} \longrightarrow \mathbb{X} \text { with } J\left(s_{1}, \ldots, s_{n}\right)=\sum_{l=1}^{n} s_{l}
$$

is a Banach isomorphism (continuous linear bijection), then we write $\mathbb{X}=S_{1} \oplus \cdots \oplus S_{n}$.

Definition 2.3 We say that a Banach nilpotent Lie group $\mathbb{M}$ of step $v$ is a Banach homogeneous group if it admits a stratification. This means there exist $v$ closed subspaces $H_{1}, \ldots, H_{v}$ such that

$$
\mathbb{M}=H_{1} \oplus \cdots \oplus H_{\nu},
$$

where $[x, y] \in H_{i+j}$ if $x \in H_{i}$ and $y \in H_{j}$ and $i+j \leq v$, and $[x, y]=0$ otherwise.

The stratification equips any Banach homogeneous group $\mathbb{M}$ with dilations $\delta_{r}: \mathbb{M} \longrightarrow \mathbb{M}$, $r>0$. These are Banach isomorphisms defined by:

$$
\delta_{r} \circ \pi_{i}=r^{i} \pi_{i},
$$

where $\pi_{i}: \mathbb{M} \rightarrow H_{i}$ is the canonical projection for all $i=1, \ldots, v$. These dilations respect the Lie bracket and Lie group structure:

$$
\left[\delta_{r} x, \delta_{r} y\right]=\delta_{r}[x, y] \quad \text { and } \quad \delta_{r}(x y)=\delta_{r}(x) \delta_{r}(y) \quad \text { for all } x, y \in \mathbb{M}, r>0 .
$$

Throughout this article, we denote by $|\cdot|$ the underlying Banach space norm on $\mathbb{M}$. It can be shown there exist positive constants $\sigma_{1}, \ldots, \sigma_{v}$ with $\sigma_{1}=1$ such that $\|\cdot\|: \mathbb{M} \rightarrow \mathbb{R}$ defined by:

$$
\|x\|=\max \left\{\sigma_{i}\left|\pi_{i}(x)\right|^{1 / i}: 1 \leq i \leq \nu\right\},
$$

satisfies the standard properties

$$
\left\|\delta_{r} x\right\|=r\|x\| \quad \text { and } \quad\|x y\| \leq\|x\|+\|y\| \quad \text { for all } x, y \in \mathbb{M}, r>0 .
$$

We say that $\|\cdot\|$ is a Banach homogeneous norm on $\mathbb{M}$. The map $\rho(x, y)=\left\|x^{-1} y\right\|$ is a distance on $\mathbb{M}$ satisfying

$$
\rho(z x, z y)=\rho(x, y) \quad \text { and } \quad \rho\left(\delta_{r} x, \delta_{r} y\right)=r \rho(x, y) \quad \text { for all } x, y, z \in \mathbb{M}, r>0 .
$$


We say that $\rho$ is a Banach homogeneous distance on $\mathbb{M}$. We also write $\rho(z)=\rho(z, 0)$ for $z \in \mathbb{M}$.

An important subclass of Banach homogeneous groups is stratified groups.

Definition 2.4 A stratified group $\mathbb{G}$ is a Banach homogeneous group for which the underlying Banach space is finite dimensional and whose first layer $V_{1}$ of the stratification $\mathbb{G}=V_{1} \oplus$ $\cdots \oplus V_{s}$ satisfies

$$
\left[V_{1}, V_{j}\right]=V_{j+1} \text { for } j \geq 1 \quad \text { and } \quad V_{j}=\{0\} \text { for } j>s .
$$

As a finite-dimensional Lie group, $\mathbb{G}$ is equipped with a Haar measure which we denote by $\mu$.

Remark 2.5 While any stratified group is also a finite-dimensional Banach homogeneous group, the converse does not hold. To see this, it suffices to consider a commutative Banach homogeneous group $\mathbb{M}=H_{1} \oplus H_{2}$, where $H_{1}$ and $H_{2}$ are finite-dimensional vector spaces. Clearly, the commutative Lie product yields $\left[H_{1}, H_{1}\right]=\{0\} \subset H_{2}$, so $\mathbb{M}$ cannot be a stratified group.

We can equip any stratified group $\mathbb{G}$ with a homogeneous distance, i.e., a distance satisfying (2.4). We define the integer:

$$
Q=\sum_{j=1}^{s} j \operatorname{dim}\left(V_{j}\right),
$$

which corresponds to the Hausdorff dimension of $\mathbb{G}$ with respect to any fixed homogeneous distance. It is a standard fact that the Haar measure $\mu$ satisfies

$$
\mu(x A)=\mu(A) \text { and } \mu\left(\delta_{r}(A)\right)=r^{Q} \mu(A) \text { for measurable } A \subset \mathbb{G}, x \in \mathbb{G} \text { and } r>0 .
$$

We consequently have

$$
\mu(B(x, r))=r^{Q} \mu(B(0,1)) \text { for every open ball } B(x, r) \subset \mathbb{G} .
$$

Fixing a basis compatible with the stratification identifies $\mathbb{G}$ with $\mathbb{R}^{n}$ for a positive integer $n$. In these coordinates, the Haar measure is simply the $n$-dimensional Lebesgue measure $\mathcal{L}^{n}$ (up to constant multiplication).

From now on, $\mathbb{M}$ will be a Banach homogeneous group with stratification $\mathbb{M}=H_{1} \oplus$ $\cdots \oplus H_{v}$ equipped with the Banach homogeneous norm $\|\cdot\|$ and corresponding homogeneous distance $\rho$. We denote by $\mathbb{G}$ a stratified group of step $s$ with stratification $\mathbb{G}=V_{1} \oplus \cdots \oplus V_{s}$ equipped with a homogeneous distance $d$. We also use the notation $d(x)=d(x, 0)$.

\subsection{Directional derivatives and differentiability}

For functions defined on general measurable domains, we may not be able to approach each point from every direction inside the domain. We will introduce an unusual but flexible definition of directional derivative, based on the following approximation of directions inside a domain.

Definition 2.6 Let $A \subset \mathbb{G}$ be measurable and $x \in \mathbb{G}$. We say that points $\zeta_{x}^{t} \in \mathbb{G}$ for $t>0$ approximate in $A$ the direction $\zeta$ at the point $x$ if

$$
x \zeta_{x}^{t} \in A \text { for every } t>0 \quad \text { and } \quad \frac{d\left(\zeta_{x}^{t}, \delta_{t} \zeta\right)}{t} \rightarrow 0 \quad \text { as } \quad t \downarrow 0 .
$$


Definition 2.7 Let $A \subset \mathbb{G}$ be a measurable set. We say that $x \in \mathbb{G}$ is a density point of $A$ if

$$
\lim _{r \downarrow 0} \frac{\mu(A \cap B(x, r))}{\mu(B(x, r))}=1 .
$$

We denote by $D(A)$ the set of density points of $A$.

Since $\mu$ is doubling, the Lebesgue differentiation theorem applies and $\mu(A \backslash D(A))=0$ for any measurable set $A \subset \mathbb{G}$. It is a standard fact that at every density point $x \in D(A)$, we have

$$
\frac{\operatorname{dist}(A, y)}{d(x, y)} \rightarrow 0 \text { as } y \rightarrow x .
$$

At density points of a measurable set, we can approximate in every direction.

Lemma 2.8 Suppose $A \subset \mathbb{G}$ is measurable and $x \in D(A)$. Then for every $\zeta \in \mathbb{G}$, there exist approximating points $\zeta_{x}^{t}$ in direction $\zeta$ at the point $x$. In addition, we can choose $\zeta_{x}^{t}$ such that the limit in (2.5) is uniform for $0<d(\zeta) \leq 1$.

Proof For each $t>0$, choose $y_{t} \in A$ such that

$$
d\left(y_{t}, x \delta_{t} \zeta\right)<\operatorname{dist}\left(A, x \delta_{t} \zeta\right)+t^{2} d(\zeta)
$$

To conclude the proof, apply (2.6) with $y=x \delta_{t} \zeta$ and define $\zeta_{x}^{t}=x^{-1} y_{t}$.

Definition 2.9 Let $A \subset \mathbb{G}$ be measurable and $f: A \rightarrow \mathbb{M}$. Suppose $x \in A$ and $\zeta \in \mathbb{G} \backslash\{0\}$ for which there exist points which approximate in $A$ the direction $\zeta$ at $x$.

We say that $f$ is differentiable at $x$ in direction $\zeta$ with directional derivative $\partial^{+} f(x, \zeta) \in$ $\mathbb{M}$ if for any choice $t \mapsto \zeta_{x}^{t}$ of points approximating in $A$ the direction $\zeta$ at $x$, we have

$$
\lim _{t \downarrow 0} \delta_{1 / t}\left(f(x)^{-1} f\left(x \zeta_{x}^{t}\right)\right)=\partial^{+} f(x, \zeta) .
$$

Proposition 2.10 Assume the hypotheses of Definition 2.9. Then, the direction $\delta_{a} \zeta$ can be approximated at $x$ for every $a>0$. Further, if $\partial^{+} f(x, \zeta)$ exists then also $\partial^{+} f\left(x, \delta_{a} \zeta\right)$ exists for every $a>0$, and we have $\partial^{+} f\left(x, \delta_{a} \zeta\right)=\delta_{a} \partial^{+} f(x, \zeta)$.

Proof Let $\zeta_{x}^{t}$ approximate the direction $\zeta$ at $x$. Then, $x \zeta_{x}^{a t} \in A$ and

$$
\frac{d\left(\zeta_{x}^{a t}, \delta_{t} \delta_{a} \zeta\right)}{t}=\frac{a d\left(\zeta_{x}^{a t}, \delta_{a t} \zeta\right)}{a t} \rightarrow 0 .
$$

Hence, $\zeta_{x}^{a t}$ approximates the direction $\delta_{a} \zeta$ at $x$.

Now, suppose $\eta_{x}^{t}$ approximates the direction $\delta_{a} \zeta$ at $x$. By the above argument, $\eta_{x}^{t / a}$ approximates the direction $\zeta$ at $x$. Using differentiability in direction $\zeta$ gives

$$
\lim _{t \downarrow 0} \delta_{1 / t}\left(f(x)^{-1} f\left(x \eta_{x}^{t}\right)\right)=\delta_{a} \lim _{t \downarrow 0} \delta_{1 / t}\left(f(x)^{-1} f\left(x \eta_{x}^{t / a}\right)\right)=\delta_{a} \partial^{+} f(x, \zeta) .
$$

Hence, $\partial^{+} f\left(x, \delta_{a} \zeta\right)=\delta_{a} \partial^{+} f(x, \zeta)$.

Remark 2.11 Let $A \subset \mathbb{G}$ be measurable, $f: A \rightarrow \mathbb{M}$ be Lipschitz and $x \in A$. Suppose there exists one curve $\zeta_{x}^{t} \in \mathbb{G}$ approximating direction $\zeta$ at $x$ with

$$
\lim _{t \downarrow 0} \delta_{1 / t}\left(f(x)^{-1} f\left(x \zeta_{x}^{t}\right)\right)=w \in \mathbb{M} .
$$


Then for any other curve $\eta_{x}^{t}$ approximating direction $\zeta$ at $x$, we also have

$$
\lim _{t \downarrow 0} \delta_{1 / t}\left(f(x)^{-1} f\left(x \eta_{x}^{t}\right)\right)=\lim _{t \downarrow 0} \delta_{1 / t}\left(f(x)^{-1} f\left(x \zeta_{x}^{t}\right)\right)=w \in \mathbb{M} .
$$

In other words, $f$ is differentiable at $x$ in direction $\zeta$ and $w=\partial^{+} f(x, \zeta)$.

Remark 2.12 In the simple case where $A \subset \mathbb{G}$ is open, we may choose the simplest curve $\zeta_{x}^{t}=\delta_{t} \zeta$ for sufficiently small $t$. Hence, by Remark 2.11 , to verify directional differentiability for a Lipschitz function $f: A \rightarrow \mathbb{M}$, we have only to check the existence of the limit:

$$
\lim _{t \downarrow 0} \delta_{1 / t}\left(f(x)^{-1} f\left(x \delta_{t} \zeta\right)\right)=\partial^{+} f(x, \zeta) .
$$

Since for Lipschitz functions directional differentiability requires only one curve $\zeta_{x}^{t}$, it is useful to find those points of the domain where we have a natural choice.

Definition 2.13 Let $A \subset \mathbb{G}$ and $x, \zeta \in \mathbb{G}$. We say that $A$ is dense at $x$ in direction $\zeta$ if

$$
\frac{\mathcal{L}^{1}\left(\left\{0<\theta<t: x \delta_{\theta} \zeta \notin A\right\}\right)}{t} \rightarrow 0 \quad \text { as } t \downarrow 0 .
$$

Proposition 2.14 Suppose $A \subset \mathbb{G}$ is measurable and dense at $x \in A$ in direction $\zeta$. Let $f: A \rightarrow \mathbb{M}$ be Lipschitz. Then, the following are true:

(1) there exist $\zeta_{x}^{t}$ approximating in $A$ the direction $\zeta$ at $x$

(2) defining $A(x, \zeta)=\left\{t \in \mathbb{R} \mid x \delta_{t} \zeta \in A\right\}$, we have $0 \in \overline{A(x, \zeta)}$

(3) $f$ is differentiable at $x$ in direction $\zeta$ if and only if the following limit exists:

$$
\lim _{t \downarrow 0, t \in A(x, \zeta)} \delta_{1 / t}\left(f(x)^{-1} f\left(x \delta_{t} \zeta\right)\right) .
$$

In either case, the above limit equals $\partial^{+} f(x, \zeta)$.

Proof For $t>0$, choose $0<T(t) \leq t$ such that $x \delta_{T(t)} \zeta \in A$ and

$$
t-T(t)<\inf \left\{t-a: 0<a \leq t, x \delta_{a} \zeta \in A\right\}+t^{2} .
$$

Density of $A$ at $x$ in direction $\zeta$ implies $T(t) / t \rightarrow 1$. The curve defined by $\zeta_{x}^{t}=\delta_{T(t)} \zeta$ then satisfies property (1).

Property (2) is clear from the definition of directional density.

Suppose now that the limit (2.7) exists. Choosing the previously defined curve $\zeta_{x}^{t}$, this gives the existence of the limit:

$$
\lim _{t \downarrow 0} \delta_{\frac{1}{t}}\left(f(x)^{-1} f\left(x \zeta_{x}^{t}\right)\right)=\lim _{t \downarrow 0} \delta_{\frac{T(t)}{t}}\left(\delta_{\frac{1}{T(t)}}\left(f(x)^{-1} f\left(x \delta_{T(t)} \zeta\right)\right)\right) .
$$

By Remark 2.11, $f$ is differentiable at $x$ in direction $\zeta$ with $\partial^{+} f(x, \zeta)$ equal to the limit in (2.7).

Conversely, assume that $f$ is differentiable at $x$ in direction $\zeta$. Considering again the previous curve $\zeta_{x}^{t}=\delta_{T(t)} \zeta$, it follows that

$$
\partial^{+} f(x, \zeta)=\lim _{t \downarrow 0, t \in A(x, \zeta)} \delta_{1 / t}\left(f(x)^{-1} f\left(x \zeta_{x}^{t}\right)\right)=\lim _{t \downarrow 0, t \in A(x, \zeta)}\left(\delta_{1 / t}\left(f(x)^{-1} f\left(x \delta_{t} \zeta\right)\right)\right) E_{x, \zeta, t},
$$

where we clearly have

$$
\rho\left(E_{x, \zeta, t}\right)=\rho\left(\delta_{1 / t}\left(f\left(x \delta_{t} \zeta\right)^{-1} f\left(x \zeta_{x}^{t}\right)\right)\right) \leq \frac{L d\left(\delta_{t} \zeta, \zeta_{x}^{t}\right)}{t} \rightarrow 0
$$

This proves property (3). 
Recall that a Banach space $X$ has the Radon-Nikodym property $(R N P)$ if every Lipschitz map $f:[0,1] \rightarrow X$ is differentiable almost everywhere. We say that a subspace of a Banach space has the RNP if it does when considered as a Banach space in its own right. To obtain directional derivatives, we use the following theorem [27, Theorem 3.1].

Theorem 2.15 Suppose $H_{1} \subset \mathbb{M}$ has the RNP. Let $A \subset \mathbb{R}$ and $\gamma: A \rightarrow \mathbb{M}$ be a Lipschitz mapping. Then, $\gamma$ is almost everywhere differentiable.

Theorem 2.16 Suppose $H_{1} \subset \mathbb{M}$ has the RNP. Let $A \subset \mathbb{G}$ be measurable and $\zeta$ be horizontal, namely $\zeta \in V_{1} \backslash\{0\}$. If $f: A \rightarrow \mathbb{M}$ is Lipschitz, then for almost every point $x \in A$ :

(1) $A$ is dense at $x$ in direction $\zeta$,

(2) $f$ is differentiable at $x$ in direction $\zeta$.

Proof Choose $\tilde{V}_{1} \subset V_{1}$ such that $\tilde{V}_{1} \oplus \operatorname{span}\{\zeta\}=V_{1}$ and consider a basis $\left(e_{1}, \ldots, e_{n-1}\right)$ of

$$
N:=\tilde{V}_{1} \oplus V_{2} \oplus \cdots \oplus V_{s},
$$

for which each $e_{i}$ belongs to some subspace $V_{j}$. The basis $\left(e_{1}, \ldots, e_{n-1}, \zeta\right)$ of $\mathbb{G}$ allows us to define $\Psi: \mathbb{R}^{n} \rightarrow \mathbb{G}$ as follows:

$$
\Psi(\xi, t)=\left(\sum_{j=1}^{n-1} \xi_{j} e_{j}\right)(t \zeta) .
$$

The map $\Psi$ is a global diffeomorphism (see [26, Proposition 7.6]). By 3.1.3(5) of [12], for a.e. $(\xi, t) \in \Psi^{-1}(A)$ the set of points

$$
\left\{\theta \in \mathbb{R}:(\xi, \theta) \notin \Psi^{-1}(A)\right\}
$$

has density zero at $t$. Since $\zeta \in V_{1}$, hence $\Psi(\xi, t)=\Psi(\xi, 0) \delta_{t} \zeta$, the previous statement implies that for a.e. $x=\Psi(\xi, t) \in A$, the set $A$ is dense at $x$ in direction $\zeta$. We denote the set of these points by $A_{\zeta}$, observing that is measurable and $\mu\left(A \backslash A_{\zeta}\right)=0$.

Let $D_{f, \zeta} \subset A_{\zeta}$ be the set of points at which $f$ is differentiable in direction $\zeta$. Our proof is completed once we have $\mu\left(A_{\zeta} \backslash D_{f, \zeta}\right)=0$, that is, $\mathcal{L}^{n}\left(\Psi^{-1}\left(A_{\zeta} \backslash D_{f, \zeta}\right)\right)$. By the measurability of $D_{f, \zeta}$ (whose proof is technical and postponed to Sect. 5.3), the set $Z_{f, \zeta}=$ $A_{\zeta} \backslash D_{f, \zeta}$ is also measurable. We can then apply Fubini's theorem to the measurable set

$$
\Psi^{-1}\left(Z_{f, \zeta}\right),
$$

whose measure can be recovered by integration of measures of the one-dimensional sections

$$
\left(\Psi^{-1}\left(Z_{f, \zeta}\right)\right)_{\xi}=\Psi(\xi, \cdot)^{-1}\left(Z_{f, \zeta}\right)
$$

where $\xi \in \mathbb{R}^{n-1}$. The composition $t \rightarrow f(\Psi(\xi, t)) \in \mathbb{M}$ is Lipschitz on $\Psi(\xi, \cdot)^{-1}\left(A_{\zeta}\right) \subset \mathbb{R}$; therefore, Theorem 2.15 provides its a.e. differentiability on $\Psi(\xi, \cdot)^{-1}\left(A_{\zeta}\right) \subset \mathbb{R}$. It follows that $\Psi(\xi, \cdot)^{-1}\left(Z_{f, \zeta}\right)$ is negligible, hence so is $\Psi^{-1}\left(Z_{f, \zeta}\right)$.

Definition 2.17 A homogeneous homomorphism, in short h-homomorphism, from $\mathbb{G}$ to $\mathbb{M}$ is a map $L: \mathbb{G} \rightarrow \mathbb{M}$ such that $L(x y)=L(x) L(y)$ and $L\left(\delta_{r}(x)\right)=\delta_{r}(L(x))$ for all $x, y \in \mathbb{G}$ and $r>0$.

Any h-homomorphism is automatically Lipschitz. Indeed, since $\mathbb{G}$ is stratified there exist a positive integer $N$ and $v_{1}, \ldots, v_{N} \in V_{1}$ such that for some $T>0$, the set

$$
V:=\left\{\delta_{t_{1}} v_{1} \cdots \delta_{t_{N}} v_{N}:\left|t_{i}\right|<T\right\}
$$


contains the unit ball. The h-homomorphism property then yields the Lipschitz continuity. The reader may also see [24, Proposition 3.11], which is stated there for stratified group targets, but works equally well for Banach group targets.

Definition 2.18 Let $A \subset \mathbb{G}$ and $x \in A \cap D(A)$. We say that $f: A \longrightarrow \mathbb{M}$ is $h$-differentiable at $x$, or simply differentiable at $x$, if there exists an h-homomorphism $L: \mathbb{G} \longrightarrow \mathbb{M}$ such that

$$
\frac{\rho\left(f(x)^{-1} f(x z), L(z)\right)}{d(z)} \rightarrow 0 \quad \text { as } z \in x^{-1} A \text { and } d(z) \downarrow 0 .
$$

The mapping $L$ is unique and called the h-differential of $f$ at $x$.

\subsection{Porous sets}

We now define porous sets and $\sigma$-porous sets.

Definition 2.19 Let $(M, \rho)$ be a metric space, $E \subset M$ and $a \in M$. We say that $E$ is porous at $a$ if there exist $\Lambda>0$ and a sequence $x_{n} \rightarrow a$ such that

$$
B\left(x_{n}, \Lambda \rho\left(a, x_{n}\right)\right) \cap E=\emptyset \quad \text { for every } n \in \mathbb{N} .
$$

A set $E$ is porous if it is porous at each point $a \in E$ with $\Lambda$ independent of $a$. A set is $\sigma$-porous if it is a countable union of porous sets.

Porous sets in stratified groups have measure zero. This follows from the fact that Haar measure on stratified groups is Ahlfors regular, hence doubling, so the Lebesgue differentiation theorem applies [19, Theorem 1.8].

\section{Directional derivatives act as h-homomorphisms outside a $\sigma$-porous set}

In this section, we study applications of porosity to directional derivatives. Recall that $\mathbb{G}$ is a stratified group of step $s$ and $\mathbb{M}$ is a Banach homogeneous group of step $v$. We will need the following estimate for the Banach homogeneous distance in $\mathbb{M}$.

Lemma 3.1 Let $N \in \mathbb{N}$ and $A_{j}, B_{j} \in \mathbb{M}$ for $j=1, \ldots, N$. Suppose there exists $b>0$ such that

$$
\rho\left(B_{j} B_{j+1} \cdots B_{N}\right) \leq b \quad \text { and } \rho\left(A_{j}, B_{j}\right) \leq b \text { for } j=1, \ldots, N .
$$

Then, there exists $C_{b}>0$, depending on $b$, such that

$$
\rho\left(A_{1} A_{2} \cdots A_{N}, B_{1} B_{2} \cdots B_{N}\right) \leq C_{b} \sum_{j=1}^{N} \rho\left(A_{j}, B_{j}\right)^{1 / v} .
$$

The finite-dimensional version of Lemma 3.1 can be found in [26, Lemma 3.7]. The proof works in a similar way for Banach homogeneous groups. However, due to the key role played by Lemma 3.1 in our arguments, we will present its proof in Sect. 5.2. Since any stratified group is a Banach homogeneous group, we can also apply Lemma 3.1 in $\mathbb{G}$, replacing $v$ by $s$ and $\rho$ by $d$.

We will also need the following estimate for distances in stratified groups [15, Lemma 2.13]. 
Lemma 3.2 There is a constant $D>0$ such that

$$
d\left(x^{-1} y x\right) \leq D\left(d(y)+d(x)^{\frac{1}{s}} d(y)^{\frac{s-1}{s}}+d(x)^{\frac{s-1}{s}} d(y)^{\frac{1}{s}}\right) \text { for } x, y \in \mathbb{G} .
$$

Unless otherwise stated, we denote by $f: A \rightarrow \mathbb{M}$ a fixed Lipschitz function on a measurable set $A \subset \mathbb{G}$ with Lipschitz constant $L$.

Definition 3.3 Fix $\zeta, \eta \in \mathbb{G}, y, z \in \mathbb{M}$ and $\varepsilon, \delta>0$. Let $C_{1}$ and $C_{2}$, respectively, be the constants obtained from applying Lemma 3.1 in $\mathbb{M}$ with $b=\max \{\varepsilon, \rho(y)+\rho(z)\}$ and in $\mathbb{G}$ with $b=\max \{\varepsilon, \varepsilon / L, d(\zeta)+d(\eta)\}$.

We define $P(\zeta, \eta, y, z, \varepsilon, \delta, A)$ to be the set of points $p \in A$ for which the following properties hold:

- For all $0<t<\delta$, there exist $\zeta_{p}^{t}, \eta_{p}^{t} \in \mathbb{G}$ satisfying

$$
\begin{aligned}
& p \zeta_{p}^{t} \in A \text { and } d\left(\zeta_{p}^{t}, \delta_{t} \zeta\right)<\varepsilon t, \\
& p \eta_{p}^{t} \in A \text { and } d\left(\eta_{p}^{t}, \delta_{t} \eta\right)<\varepsilon t, \\
& \rho\left(f(p)^{-1} f\left(p \zeta_{p}^{t}\right), \delta_{t} y\right) \leq \varepsilon t, \\
& \rho\left(f(p)^{-1} f\left(p \eta_{p}^{t}\right), \delta_{t} z\right) \leq \varepsilon t .
\end{aligned}
$$

- For arbitrarily small $t$, there exist $\omega_{p}^{t} \in \mathbb{G}$ such that

$$
\begin{gathered}
p \omega_{p}^{t} \in A \text { and } d\left(\omega_{p}^{t}, \delta_{t}(\zeta \eta)\right)<\varepsilon t \\
\rho\left(f(p)^{-1} f\left(p \omega_{p}^{t}\right), \delta_{t}(y z)\right)>\left(3 C_{1} \varepsilon^{1 / v}+L \varepsilon+L C_{2}\left(2+L^{-1 / s}\right) \varepsilon^{1 / s}\right) t .
\end{gathered}
$$

Intuitively, $P(\zeta, \eta, y, z, \varepsilon, \delta, A)$ consists of points for which the directional derivatives in direction $\zeta$ and $\eta$ look like $y$ and $z$, respectively, at scales less than $\delta$ and with accuracy $\varepsilon$.

Notice that provided $\varepsilon$ and $\delta$ are bounded by some fixed constant $K$, the constants $C_{1}$ and $C_{2}$ in Definition 3.3 are bounded by constants independent of the precise value of $\varepsilon$ and $\delta$.

Lemma 3.4 The set $P(\zeta, \eta, y, z, \varepsilon, \delta, A)$ is porous.

Proof For this proof, we abbreviate $P=P(\zeta, \eta, y, z, \varepsilon, \delta, A)$. Let $x \in P$ and choose $0<t<\delta / 2$ for which there exist $\omega_{x}^{t} \in \mathbb{G}$ satisfying (3.6) and (3.7). Fix also $\zeta_{x}^{t}$ satisfying (3.2) and (3.4). Since $x$ was any element of $P$ and $t$ could be chosen arbitrarily small, to prove $P$ is porous it suffices to show that $B\left(x \zeta_{x}^{t}, \varepsilon t / L\right) \cap P=\emptyset$.

We suppose $B\left(x \zeta_{x}^{t}, \varepsilon t / L\right) \cap P \neq \varnothing$ and deduce a contradiction. Choose $p \in$ $B\left(x \zeta_{x}^{t}, \varepsilon t / L\right) \cap P$. Use the definition of $P$ to choose $\eta_{p}^{t} \in \mathbb{G}$ satisfying (3.3) and (3.5). We first estimate

$$
\rho\left(f(x)^{-1} f\left(p \eta_{p}^{t}\right), \delta_{t}(y z)\right) .
$$

To this end, observe that we can write:

$$
\delta_{1 / t}\left(f(x)^{-1} f\left(p \eta_{p}^{t}\right)\right)=A_{1} A_{2} A_{3}, \quad y z=B_{1} B_{2} B_{3},
$$

where

$$
\begin{aligned}
& A_{1}=\delta_{1 / t}\left(f(x)^{-1} f\left(x \zeta_{x}^{t}\right)\right), \quad A_{2}=\delta_{1 / t}\left(f\left(x \zeta_{x}^{t}\right)^{-1} f(p)\right), \\
& A_{3}=\delta_{1 / t}\left(f(p)^{-1} f\left(p \eta_{p}^{t}\right)\right)
\end{aligned}
$$

and

$$
B_{1}=y, \quad B_{2}=0, \quad B_{3}=z .
$$


We check $A_{i}, B_{i}$ satisfy the hypotheses of Lemma 3.1 with $b=\max \{\varepsilon, \rho(y)+\rho(z)\}$. Using (3.4) gives

$$
\rho\left(A_{1}, B_{1}\right)=\rho\left(\delta_{1 / t}\left(f(x)^{-1} f\left(x \zeta_{x}^{t}\right)\right), y\right)=\frac{1}{t} \rho\left(f(x)^{-1} f\left(x \zeta_{x}^{t}\right), \delta_{t} y\right) \leq \varepsilon .
$$

Recalling that $p \in B\left(x \zeta_{x}^{t}, \varepsilon t / L\right)$, we obtain

$$
\rho\left(A_{2}, B_{2}\right)=\rho\left(\delta_{1 / t}\left(f\left(x \zeta_{x}^{t}\right)^{-1} f(p)\right), 0\right)=\frac{1}{t} \rho\left(f(p), f\left(x \zeta_{x}^{t}\right)\right) \leq \frac{L}{t} d\left(x \zeta_{x}^{t}, p\right) \leq \varepsilon .
$$

Using (3.5) also gives

$$
\rho\left(A_{3}, B_{3}\right)=\rho\left(\delta_{1 / t}\left(f(p)^{-1} f\left(p \eta_{p}^{t}\right)\right), z\right)=\frac{1}{t} \rho\left(f(p)^{-1} f\left(p \eta_{p}^{t}\right), \delta_{t} z\right) \leq \varepsilon .
$$

Hence, $\rho\left(A_{i}, B_{i}\right) \leq \varepsilon$ for each $i$. Clearly, also $\rho\left(B_{i} B_{i+1} \cdots B_{3}\right) \leq \rho(y)+\rho(z)$ for each $i$. Hence, the hypotheses of Lemma 3.1 are satisfied for our choice of $b$, and we get

$$
\rho\left(f(x)^{-1} f\left(p \eta_{p}^{t}\right), \delta_{t}(y z)\right) \leq C_{1} t \sum_{i=1}^{3} d\left(A_{i}, B_{i}\right)^{1 / v} \leq 3 C_{1} \varepsilon^{1 / v} t .
$$

Now, let $\theta_{x}^{t}=x^{-1} p \eta_{p}^{t}$. Then, $x \theta_{x}^{t}=p \eta_{p}^{t} \in A$ and (3.8) gives

$$
\rho\left(f(x)^{-1} f\left(x \theta_{x}^{t}\right), \delta_{t}(y z)\right) \leq 3 C_{1} \varepsilon^{1 / v} t .
$$

Now, we estimate $d\left(\theta_{x}^{t}, \delta_{t}(\zeta \eta)\right)$. Since $p \in B\left(x \zeta_{x}^{t}, \varepsilon t / L\right)$, we may choose $h^{t} \in \mathbb{G}$ with $d\left(h^{t}\right) \leq \varepsilon t / L$ and $p=x \zeta_{x}^{t} h^{t}$. Therefore

$$
d\left(\theta_{x}^{t}, \delta_{t}(\zeta \eta)\right)=d\left(x^{-1} p \eta_{p}^{t}, \delta_{t}(\zeta \eta)\right)=d\left(\zeta_{x}^{t} h^{t} \eta_{p}^{t}, \delta_{t}(\zeta \eta)\right) .
$$

Now, define

$$
A_{1}=\delta_{1 / t} \zeta_{x}^{t}, \quad A_{2}=\delta_{1 / t} h^{t}, \quad A_{3}=\delta_{1 / t} \eta_{p}^{t},
$$

and

$$
B_{1}=\zeta, \quad B_{2}=0, \quad B_{3}=\eta .
$$

Let $b=\max \{\varepsilon, \varepsilon / L, d(\zeta)+d(\eta)\}$. Using (3.2) and (3.3) shows $d\left(A_{1}, B_{1}\right)$ and $d\left(A_{3}, B_{3}\right)$ are bounded by $\varepsilon$. Using $d\left(h^{t}\right) \leq \varepsilon t / L$ gives $d\left(A_{2}, B_{2}\right) \leq \varepsilon / L$. Clearly, $d\left(B_{i} B_{i+1} \cdots B_{3}\right) \leq$ $d(\zeta)+d(\eta)$ for each $i$. Hence, the hypotheses of Lemma 3.1 are satisfied, giving

$$
d\left(\theta_{x}^{t}, \delta_{t}(\zeta \eta)\right) \leq C_{2} t \sum_{i=1}^{3} \rho\left(A_{i}, B_{i}\right)^{1 / s} \leq C_{2}\left(2+L^{-1 / s}\right) \varepsilon^{1 / s} t .
$$

Recall that $\omega_{x}^{t}$ were chosen satisfying (3.6) and (3.7). Using also (3.9), we estimate as follows:

$$
\begin{aligned}
\rho\left(f(x)^{-1} f\left(x \omega_{x}^{t}\right), \delta_{t}(y z)\right) & \leq \rho\left(f(x)^{-1} f\left(x \theta_{x}^{t}\right), \delta_{t}(y z)\right)+\rho\left(f\left(x \omega_{x}^{t}\right), f\left(x \theta_{x}^{t}\right)\right) \\
& \leq 3 C_{1} \varepsilon^{1 / v} t+L d\left(\omega_{x}^{t}, \theta_{x}^{t}\right) \\
& \leq 3 C_{1} \varepsilon^{1 / v} t+L\left(d\left(\omega_{x}^{t}, \delta_{t}(\zeta \eta)\right)+d\left(\theta_{x}^{t}, \delta_{t}(\zeta \eta)\right)\right) \\
& \leq 3 C_{1} \varepsilon^{1 / v} t+L \varepsilon t+L C_{2}\left(2+L^{-1 / s}\right) \varepsilon^{1 / s} t \\
& =\left(3 C_{1} \varepsilon^{1 / v}+L \varepsilon+L C_{2}\left(2+L^{-1 / s}\right) \varepsilon^{1 / s}\right) t .
\end{aligned}
$$

This contradicts the choice of $\omega_{x}^{t}$, forcing us to conclude $B\left(x \zeta_{x}^{t}, \varepsilon t / L\right) \cap P=\emptyset$. Hence, $P$ is porous, which concludes the proof. 
We now prove Theorem 1.2 by putting together countably many of the sets from Definition 3.3 .

Proof of Theorem 1.2 Using Theorem 5.2, we can assume that the Banach homogeneous group $\mathbb{M}$ is separable. Let $P$ be the countable union of sets $P(\zeta, \eta, y, z, \varepsilon, \delta, A)$ for $\zeta, \eta$ in a countable dense subset $\mathcal{N}_{\mathbb{G}}$ of $\mathbb{G}, y, z$ in a countable dense subset $\mathcal{N}_{\mathbb{M}}$ of $\mathbb{M}$, and $\varepsilon, \delta$ positive rationals. By Proposition 2.10, it is enough to show that for points $x \in A \cap D(A) \backslash P$, we have

$$
\partial^{+} f(x, \zeta \eta)=\partial^{+} f(x, \zeta) \partial^{+} f(x, \eta)
$$

whenever $\zeta, \eta \in \mathbb{G}$ for which $\partial^{+} f(x, \zeta), \partial^{+} f(x, \eta)$ exist.

Suppose $x \in A \cap D(A) \backslash P$ and $\partial^{+} f(x, \zeta), \partial^{+} f(x, \eta)$ exist for some $\zeta, \eta \in \mathbb{G}$. Fix $\varepsilon>0$ rational. Choose $\zeta_{x}^{t}, \eta_{x}^{t} \in \mathbb{G}$ for $t>0$ with $x \zeta_{x}^{t}, x \eta_{x}^{t} \in A$ and $d\left(\zeta_{x}^{t}, \delta_{t} \zeta\right) / t, d\left(\eta_{x}^{t}, \delta_{t} \eta\right) / t \rightarrow 0$. Using the existence of $\partial^{+} f(x, \zeta), \partial^{+} f(x, \eta)$, choose $\delta>0$ rational such that for $0<t<\delta$ :

$$
\begin{aligned}
& d\left(\zeta_{x}^{t}, \delta_{t} \zeta\right)<\varepsilon t / 2 \text { and } \rho\left(\delta_{1 / t}\left(f(x)^{-1} f\left(x \zeta_{x}^{t}\right)\right), \partial^{+} f(x, \zeta)\right) \leq \varepsilon / 2, \\
& d\left(\eta_{x}^{t}, \delta_{t} \eta\right)<\varepsilon t / 2 \text { and } \rho\left(\delta_{1 / t}\left(f(x)^{-1} f\left(x \eta_{x}^{t}\right)\right), \partial^{+} f(x, \eta)\right) \leq \varepsilon / 2 .
\end{aligned}
$$

Now, choose $y, z \in \mathcal{N}_{\mathbb{M}}$ such that

$$
\rho\left(\partial^{+} f(x, \zeta), y\right)<\varepsilon / 2, \quad \rho\left(\partial^{+} f(x, \eta), z\right)<\varepsilon / 2, \quad \rho\left(\partial^{+} f(x, \zeta) \partial^{+} f(x, \eta), y z\right)<C_{1} \varepsilon^{1 / \nu} .
$$

In particular, we have

$$
\rho\left(\delta_{1 / t}\left(f(x)^{-1} f\left(x \zeta_{x}^{t}\right)\right), y\right) \leq \varepsilon \quad \text { and } \quad \rho\left(\delta_{1 / t}\left(f(x)^{-1} f\left(x \eta_{x}^{t}\right)\right), z\right) \leq \varepsilon \quad \text { for } 0<t<\delta .
$$

Now, choose $\zeta^{\prime}, \eta^{\prime} \in \mathcal{N}_{\mathbb{G}}$ such that $d\left(\zeta, \zeta^{\prime}\right)<\varepsilon / 2, d\left(\eta, \eta^{\prime}\right)<\varepsilon / 2$ and $d\left(\zeta \eta, \zeta^{\prime} \eta^{\prime}\right)<\varepsilon / 2$. Then,

$$
d\left(\zeta_{x}^{t}, \delta_{t} \zeta^{\prime}\right)<\varepsilon t \text { and } d\left(\eta_{x}^{t}, \delta_{t} \eta^{\prime}\right)<\varepsilon t \quad \text { for } 0<t<\delta .
$$

Equations (3.11) and (3.12) show that the point $x$ satisfies the first four conditions of the set $P\left(\zeta^{\prime}, \eta^{\prime}, y, z, \varepsilon, \delta, A\right)$, i.e., (3.2)-(3.5) hold with $\zeta, \eta$ replaced by $\zeta^{\prime}, \eta^{\prime}$ and $p$ replaced by $x$.

Since $x \notin P \supset P\left(\zeta^{\prime}, \eta^{\prime}, y, z, \varepsilon, \delta, A\right)$, the analogue of (3.6) or (3.7) for the set $P\left(\zeta^{\prime}, \eta^{\prime}, y, z, \varepsilon, \delta, A\right)$ must fail for sufficiently small $t$. Hence, whenever $t$ is sufficiently small and $\omega_{x}^{t}$ satisfy the two conditions

$$
x \omega_{x}^{t} \in A \quad \text { and } \quad d\left(\omega_{x}^{t}, \delta_{t}\left(\zeta^{\prime} \eta^{\prime}\right)\right)<\varepsilon t
$$

then we must have

$$
\rho\left(f(x)^{-1} f\left(x \omega_{x}^{t}\right), \delta_{t}(y z)\right) \leq\left(3 C_{1} \varepsilon^{1 / v}+L \varepsilon+L C_{2}\left(2+L^{-1 / s}\right) \varepsilon^{1 / s}\right) t .
$$

Let $\omega_{x}^{t}$ approximate in $A$ the direction $\zeta \eta$ at $x$. Then $x \omega_{x}^{t} \in A$ and $d\left(\omega_{x}^{t}, \delta_{t}(\zeta \eta)\right)<\varepsilon t / 2$ for sufficiently small $t$. Since $d\left(\zeta \eta, \zeta^{\prime} \eta^{\prime}\right)<\varepsilon / 2$, it follows that $\omega_{x}^{t}$ satisfy (3.13) and hence (3.14) for sufficiently small $t$. Taking into account the third inequality of (3.10), we obtain for sufficiently small $t$ :

$\rho\left(f(x)^{-1} f\left(x \omega_{x}^{t}\right), \delta_{t}\left(\partial^{+} f(x, \zeta) \partial^{+} f(x, \eta)\right)\right) \leq\left(4 C_{1} \varepsilon^{1 / v}+L \varepsilon+L C_{2}\left(2+L^{-1 / s}\right) \varepsilon^{1 / s}\right) t$.

To summarize, if $\omega_{x}^{t}$ approximate the direction $\zeta \eta$ at $x$ then (3.15) holds for sufficiently small $t$. Provided $\varepsilon, \delta<1$, the constants $C_{1}$ and $C_{2}$ are independent of the precise value of $\varepsilon$ and $\delta$. We conclude that $\partial^{+} f(x, \zeta \eta)$ exists and is equal to $\partial^{+} f(x, \zeta) \partial^{+} f(x, \eta)$. 


\section{From directional derivatives to differentiability}

We now prove Theorem 1.3 which gives conditions for pointwise differentiability. Combining these results with those of Sect. 3 will yield Theorem 1.1.

Proof of Theorem 1.3 First, we suppose $f$ is differentiable at $x \in A \cap D(A)$. Then, there exists an h-homomorphism $L_{x}: \mathbb{G} \rightarrow \mathbb{M}$ such that

$$
\rho\left(f(x)^{-1} f(x z), L_{x}(z)\right)=o(d(z)) \quad \text { as } z \in x^{-1} A \quad \text { and } \quad z \rightarrow 0 .
$$

Let $\zeta \in \mathbb{G}$. Using Lemma 2.8, choose $\zeta_{x}^{t}$ such that $x \zeta_{x}^{t} \in A$ and $d\left(\zeta_{x}^{t}, \delta_{t} \zeta\right) / t \rightarrow 0$ as $t \downarrow 0$. Then, (4.1) gives

$$
\rho\left(f(x)^{-1} f\left(x \zeta_{x}^{t}\right), L_{x}\left(\zeta_{x}^{t}\right)\right)=o\left(d\left(\zeta_{x}^{t}\right)\right) \quad \text { as } t \downarrow 0 .
$$

Since $d\left(\zeta_{x}^{t}, \delta_{t} \zeta\right) / t \rightarrow 0$, there exists $K=K(\zeta)>0$ such that $d\left(\zeta_{x}^{t}\right)<K t$ for sufficiently small $t$. Combining this with (4.2) gives

$$
\lim _{t \downarrow 0} \rho\left(\delta_{1 / t}\left(f(x)^{-1} f\left(x \zeta_{x}^{t}\right)\right), \delta_{1 / t} L_{x}\left(\zeta_{x}^{t}\right)\right)=0 .
$$

Since $L_{x}$ is an h-homomorphism (and hence Lipschitz), we have

$$
\rho\left(L_{x}(\zeta), \delta_{1 / t} L_{x}\left(\zeta_{x}^{t}\right)\right)=\rho\left(L_{x}(\zeta), L_{x}\left(\delta_{1 / t} \zeta_{x}^{t}\right)\right) \leq \operatorname{Lip}\left(L_{x}\right) \frac{d\left(\delta_{t} \zeta, \zeta_{x}^{t}\right)}{t} \rightarrow 0 .
$$

Combining this with (4.3) shows $\partial^{+} f(x, \zeta)$ exists and is equal to $L_{x}(\zeta)$ for any $\zeta \in \mathbb{G}$. Since $L_{x}$ is an h-homomorphism, the h-homomorphism property of directional derivatives follows.

For the converse statement, we assume $\partial^{+} f(x, \zeta)$ exists for any $\zeta \in \mathbb{G}$ and $L_{x}: \mathbb{G} \rightarrow \mathbb{M}$ defined by $L_{x}(\zeta):=\partial^{+} f(x, \zeta)$ is an h-homomorphism. For every $\zeta \in \mathbb{G}$ with $d(\zeta) \leq 1$, use Lemma 2.8 to choose $\zeta_{x}^{t}$ for $t>0$ with $x \zeta_{x}^{t} \in A$ and $d\left(\zeta_{x}^{t}, \delta_{t} \zeta\right) / t \rightarrow 0$ as $t \downarrow 0$ uniformly for $\zeta \in \mathbb{G}$ with $d(\zeta) \leq 1$. We first show that

$$
\delta_{1 / t}\left(f(x)^{-1} f\left(x \zeta_{x}^{t}\right)\right) \rightarrow \partial^{+} f(x, \zeta) \quad \text { as } t \downarrow 0 \text { uniformly for } \zeta \in \mathbb{G} \text { with } d(\zeta) \leq 1 \text {. }
$$

Let $K=1+\operatorname{Lip}\left(L_{x}\right)+3 L>0$ and fix $\varepsilon>0$. Choose a finite set $S \subset \mathbb{G}$ such that for any $\eta \in \mathbb{G}$ with $d(\eta) \leq 1$, there exists $\zeta \in S$ with $d(\zeta, \eta)<\varepsilon / K$. Choose $\delta>0$ such that

$$
\rho\left(\delta_{1 / t}\left(f(x)^{-1} f\left(x \zeta_{x}^{t}\right)\right), \partial^{+} f(x, \zeta)\right)<\varepsilon / K \quad \text { for every } \zeta \in S \text { and } 0<t<\delta,
$$

and

$$
d\left(\eta_{x}^{t}, \delta_{t} \eta\right) / t<\varepsilon / K \quad \text { for every } \eta \in \mathbb{G} \text { with } d(\eta) \leq 1 \text { and } 0<t<\delta .
$$

Given $\eta \in \mathbb{G}$ with $d(\eta) \leq 1$, choose $\zeta \in S$ with $d(\zeta, \eta)<\varepsilon / K$. We observe that

$$
\begin{aligned}
\rho\left(\delta_{1 / t}\left(f(x)^{-1} f\left(x \eta_{x}^{t}\right)\right), \partial^{+} f(x, \eta)\right) \leq & \rho\left(\delta_{1 / t}\left(f(x)^{-1} f\left(x \zeta_{x}^{t}\right)\right), \partial^{+} f(x, \zeta)\right) \\
& +\rho\left(\delta_{1 / t}\left(f(x)^{-1} f\left(x \eta_{x}^{t}\right)\right), \delta_{1 / t}\left(f(x)^{-1} f\left(x \zeta_{x}^{t}\right)\right)\right) \\
& +\rho\left(\partial^{+} f(x, \zeta), \partial^{+} f(x, \eta)\right) .
\end{aligned}
$$

The first term in (4.7) is estimated by (4.5) for $0<t<\delta$. We estimate the second term of (4.7) as follows:

$\rho\left(\delta_{1 / t}\left(f(x)^{-1} f\left(x \eta_{x}^{t}\right)\right), \delta_{1 / t}\left(f(x)^{-1} f\left(x \zeta_{x}^{t}\right)\right)\right)=\frac{1}{t} \rho\left(f\left(x \eta_{x}^{t}\right), f\left(x \zeta_{x}^{t}\right)\right)$ 


$$
\begin{aligned}
& \leq \frac{L}{t} d\left(\eta_{x}^{t}, \zeta_{x}^{t}\right) \\
& \leq L\left(\frac{d\left(\eta_{x}^{t}, \delta_{t} \eta\right)}{t}+\frac{d\left(\zeta_{x}^{t}, \delta_{t} \zeta\right)}{t}+d(\zeta, \eta)\right)
\end{aligned}
$$

Using our choice of $\zeta$ and (4.6), we obtain

$$
\rho\left(\delta_{1 / t}\left(f(x)^{-1} f\left(x \eta_{x}^{t}\right)\right), \delta_{1 / t}\left(f(x)^{-1} f\left(x \zeta_{x}^{t}\right)\right)\right)<3 L \varepsilon / K \quad \text { for } 0<t<\delta .
$$

We estimate the final term in (4.7) as follows:

$$
\rho\left(\partial^{+} f(x, \zeta), \partial^{+} f(x, \eta)\right)=\rho\left(L_{x}(\zeta), L_{x}(\eta)\right) \leq \operatorname{Lip}\left(L_{x}\right) d(\zeta, \eta)<\varepsilon \operatorname{Lip}\left(L_{x}\right) / K .
$$

Using (4.5), (4.8) and (4.9) in (4.7) together with the definition of $K$ yields

$$
\rho\left(\delta_{1 / t}\left(f(x)^{-1} f\left(x \eta_{x}^{t}\right)\right), \partial^{+} f(x, \eta)\right)<\varepsilon .
$$

This proves the uniform convergence (4.4).

To conclude, we will show that $\rho\left(f(x)^{-1} f(x z), L_{x}(z)\right)=o(d(z))$ as $z \rightarrow 0$ with $z \in x^{-1} A$. First, notice that for $\zeta \in \mathbb{G}$ with $d(\zeta) \leq 1$ :

$$
\begin{aligned}
\frac{1}{t} \rho\left(f(x)^{-1} f\left(x \zeta_{x}^{t}\right), L_{x}\left(\zeta_{x}^{t}\right)\right)= & \rho\left(\delta_{1 / t}\left(f(x)^{-1} f\left(x \zeta_{x}^{t}\right)\right), \delta_{1 / t} L_{x}\left(\zeta_{x}^{t}\right)\right) \\
\leq & \rho\left(\delta_{1 / t}\left(f(x)^{-1} f\left(x \zeta_{x}^{t}\right)\right), \partial^{+} f(x, \zeta)\right) \\
& +\rho\left(\partial^{+} f(x, \zeta), \delta_{1 / t} L_{x}\left(\zeta_{x}^{t}\right)\right) \\
= & \rho\left(\delta_{1 / t}\left(f(x)^{-1} f\left(x \zeta_{x}^{t}\right)\right), \partial^{+} f(x, \zeta)\right)+\rho\left(L_{x}(\zeta), L_{x}\left(\delta_{1 / t} \zeta_{x}^{t}\right)\right) \\
\leq & \rho\left(\delta_{1 / t}\left(f(x)^{-1} f\left(x \zeta_{x}^{t}\right)\right), \partial^{+} f(x, \zeta)\right)+\operatorname{Lip}\left(L_{x}\right) d\left(\zeta, \delta_{1 / t} \zeta_{x}^{t}\right) .
\end{aligned}
$$

Hence (4.4), and our choice of $\zeta_{x}^{t}$ gives

$$
\frac{1}{t} \rho\left(f(x)^{-1} f\left(x \zeta_{x}^{t}\right), L_{x}\left(\zeta_{x}^{t}\right)\right) \rightarrow 0 \quad \text { as } t \downarrow 0 \text { uniformly for } d(\zeta) \leq 1 .
$$

Given $\varepsilon>0$, use (4.10) and our choice of $\zeta_{x}^{t}$ to choose $\delta>0$ such that

$$
\frac{d\left(\delta_{t} \zeta, \zeta_{x}^{t}\right)}{t}<\varepsilon \text { and } \frac{\rho\left(f(x)^{-1} f\left(x \zeta_{x}^{t}\right), L_{x}\left(\zeta_{x}^{t}\right)\right)}{t}<\varepsilon \quad \text { for } d(\zeta) \leq 1 \text { and } 0<t<\delta .
$$

Now, let $z \in x^{-1} A$ with $d(z) \leq \delta$. Choose $\zeta \in \mathbb{G}$ with $d(\zeta)=1$ such that $z=\delta_{t} \zeta$ for some $0<t<\delta$. We then estimate as follows:

$$
\begin{aligned}
\frac{\rho\left(f(x)^{-1} f(x z), L_{x}(z)\right)}{d(z)} \leq & \frac{\rho\left(f(x)^{-1} f\left(x \delta_{t} \zeta\right), f(x)^{-1} f\left(x \zeta_{x}^{t}\right)\right)}{t} \\
& +\frac{\rho\left(f(x)^{-1} f\left(x \zeta_{x}^{t}\right), L_{x}\left(\zeta_{x}^{t}\right)\right)}{t} \\
& +\frac{\rho\left(L_{x}\left(\zeta_{x}^{t}\right), L_{x}\left(\delta_{t} \zeta\right)\right)}{t} \\
\leq & \left(L+\operatorname{Lip}\left(L_{x}\right)\right) \frac{d\left(\delta_{t} \zeta, \zeta_{x}^{t}\right)}{t}+\frac{\rho\left(f(x)^{-1} f\left(x \zeta_{x}^{t}\right), L_{x}\left(\zeta_{x}^{t}\right)\right)}{t} \\
& <\left(L+\operatorname{Lip}\left(L_{x}\right)+1\right) \varepsilon .
\end{aligned}
$$

This shows that $f$ is differentiable at $x$. 
Using our results above, we can now quickly show how Theorem 1.3 gives Theorem 1.1. By [26, Lemma 4.9], there exists a spanning set of directions $v_{1}, \ldots, v_{N} \in V_{1}$ for some $N \in \mathbb{N}$; namely, there exists $T>0$ such that

$$
\left\{\delta_{t_{1}} v_{1} \delta_{t_{2}} v_{2} \cdots \delta_{t_{N}} v_{N}: 0 \leq t_{i}<T\right\}
$$

contains the unit ball of $\mathbb{G}$. If $H_{1} \subset \mathbb{M}$ has the RNP, then Theorem 2.16 yields a null set $Z \subset A$ such that for every $x \in A \backslash Z: A$ is dense at $x$ in direction $v_{i}$ and $\partial^{+} f\left(x, v_{i}\right)$ exists for every $i=1, \ldots, N$. By Theorem 1.2, we can find a $\sigma$-porous set $P \subset \mathbb{G}$ such that directional derivatives at $x$ act as h-homomorphisms whenever $x \in A \cap D(A) \backslash P$.

Combining these with the spanning property of $v_{1}, \ldots, v_{N}$, it follows that $f$ is differentiable at each $x \in A \cap D(A) \backslash(P \cup Z)$ in any direction $\zeta$ of the unit ball. Proposition 2.10 extends this directional differentiability to all directions $\zeta$ of $\mathbb{G}$. The same Theorem 1.2 shows that for $x \in A \cap D(A) \backslash(P \cup Z)$, the directional derivative $\mathbb{G} \ni \zeta \rightarrow \partial^{+} f(x, \zeta)$ defines an h-homomorphism. We have established both conditions 1 and 2 of Theorem 1.3; therefore, $f$ is differentiable at every point of $A \cap D(A) \backslash(P \cup Z)$. Since $\mathcal{H}_{d}^{Q}(A \backslash D(A))=0$ and porous sets have measure zero, our claim follows.

Remark 4.1 Taking into account Remark 2.12, when the domain $A$ of $f$ is an open set, properties (3.2) and (3.3) of Definition 3.3 are automatically satisfied. Hence, several arguments in the proofs of both Theorem 1.2 and Theorem 1.3 become simpler.

Acknowledgements The second and third named authors thank the organizers of the workshop "Singular Phenomena and Singular Geometries," Pisa, June 20-23, 2016, for the warm hospitality and for the scientific environment, where the project of the present paper started.

\section{Appendix}

\subsection{Separable Banach homogeneous groups}

Let $\mathbb{M}$ be a Banach homogeneous group of step $v$. For every $\mathfrak{v}=\left(v_{1}, \ldots, v_{k}\right) \in \mathbb{M}^{k}, k \geq 1$, we define the nonassociative product:

$$
\left.\mathfrak{p}_{k}(\mathfrak{v})=\left[\cdots\left[\left[v_{1}, v_{2}\right], v_{3}\right] \cdots\right], v_{k}\right]=v_{1} \circ v_{2} \circ \cdots \circ v_{k},
$$

where $\mathfrak{p}_{k}: \mathbb{M}^{k} \rightarrow \mathbb{M}$ is clearly a continuous multilinear mapping. For any countable subset

$$
\mathcal{N}=\left\{x_{j} \in \mathbb{M}: j \in \mathbb{N}\right\},
$$

we define its rational homogeneous span as follows:

$$
\langle\langle\mathcal{N}\rangle\rangle=\left\{\sum_{j=0}^{n} \sum_{l=1}^{v} \lambda_{j l} \pi_{l}\left(x_{j}\right): \lambda_{j l} \in \mathbb{Q}, n \in \mathbb{N}\right\} \subset \mathbb{M} .
$$

The closed homogeneous span of $\mathcal{N}$ is simply $\langle\mathcal{N}\rangle=\overline{\langle\langle\mathcal{N}\rangle\rangle} \subset \mathbb{M}$. This is clearly a linear subspace of $\mathbb{M}$ that is also homogeneous, that is, $\delta_{r}\langle\mathcal{N}\rangle \subset\langle\mathcal{N}\rangle$ for every $r>0$. Since $\langle\langle\mathcal{N}\rangle\rangle$ is countable, $\langle\mathcal{N}\rangle$ is a separable homogeneous Banach space that in particular contains $\overline{\mathcal{N}}$.

Theorem 5.1 There exists a separable Banach homogeneous subgroup $\mathbb{V} \subset \mathbb{M}$ such that $\langle\mathcal{N}\rangle \subset \mathbb{V}$. 
Proof Let $\mathrm{Y}_{1}=\langle\mathcal{N}\rangle$ be the separable and homogeneous Banach space spanned by $\mathcal{N}$, and consider

$$
\mathbf{Y}_{k}=\left\{\sum_{\mathfrak{v} \in \mathcal{I}} \mathfrak{p}_{k}(\mathfrak{v}): \mathcal{I} \subset \mathbf{Y}_{1}^{k} \text { is finite }\right\}
$$

for each $k=2, \ldots, v$. Since dilations are also Lie algebra homomorphisms and $\mathbf{Y}_{1}$ is closed under dilations, it follows that $\delta_{r} \mathbf{Y}_{k} \subset \mathbf{Y}_{k}$ for each $k=1, \ldots, v$ and $r>0$. It is clear that $\mathbf{Y}_{k}$ is a linear subspace also for $k=2, \ldots, v$.

We wish to show that for any couple of finite subsets $\mathcal{I} \subset \mathrm{Y}_{1}^{k}$ and $\mathcal{J} \subset \mathrm{Y}_{1}^{l}$ with

$$
\sum_{\mathfrak{v} \in \mathcal{I}} \mathfrak{p}_{k}(\mathfrak{v}) \in \mathbf{Y}_{k} \text { and } \sum_{\mathfrak{w} \in \mathcal{J}} \mathfrak{p}_{l}(\mathfrak{w}) \in \mathbf{Y}_{l}
$$

the following conditions hold

$$
\left[y, \sum_{\mathfrak{w} \in \mathcal{J}} \mathfrak{p}_{l}(\mathfrak{w})\right] \in \mathbf{Y}_{l+1} \text { and }\left[\sum_{\mathfrak{v} \in \mathcal{I}} \mathfrak{p}_{k}(\mathfrak{v}), \sum_{\mathfrak{w} \in \mathcal{J}} \mathfrak{p}_{l}(\mathfrak{w})\right] \in \mathbf{Y}_{k+l}
$$

where $y \in \mathbb{Y}_{1}$. Notice that the nilpotence of $\mathbb{M}$ gives $\mathbf{Y}_{k+l}=\{0\}$ whenever $k+l>v$. The first condition of (5.1) is straightforward:

$$
\left[y, \sum_{\mathfrak{v} \in \mathcal{J}} \mathfrak{p}_{l}(\mathfrak{w})\right]=-\sum_{\mathfrak{v} \in \mathcal{J}} \mathfrak{p}_{l+1}((\mathfrak{w}, y)) \in \mathbf{Y}_{l+1} .
$$

The second one for $k=2$ is a consequence of the Jacobi identity that yields

$$
\begin{aligned}
{\left[\left[v_{1}, v_{2}\right], \sum_{\mathfrak{w} \in \mathcal{J}} \mathfrak{p}_{l}(\mathfrak{w})\right] } & =-\left[\left[v_{2}, \sum_{\mathfrak{w} \in \mathcal{J}} \mathfrak{p}_{l}(\mathfrak{w})\right], v_{1}\right]-\left[\left[\sum_{\mathfrak{w} \in \mathcal{J}} \mathfrak{p}_{l}(\mathfrak{w}), v_{1}\right], v_{2}\right] \\
& =\sum_{\mathfrak{w} \in \mathcal{J}} \mathfrak{p}_{l+2}\left(\left(\mathfrak{w}, v_{2}, v_{1}\right)\right)-\sum_{\mathfrak{w} \in \mathcal{J}} \mathfrak{p}_{l+2}\left(\left(\mathfrak{w}, v_{1}, v_{2}\right)\right) \in \mathbb{Y}_{l+2}
\end{aligned}
$$

for every positive integer $l$. If we assume the second condition of (5.1) to hold for a fixed $k \geq 2$ and every $l \geq 1$, then setting

$$
\mathfrak{v}=\left(v_{1}, v_{2}, \ldots, v_{k+1}\right) \in \mathbf{Y}_{1}^{k+1} \text { and } \tilde{\mathfrak{v}}=\left(v_{1}, v_{2}, \ldots, v_{k}\right) \in \mathbf{Y}_{1}^{k},
$$

we consider the product

$$
\begin{aligned}
{\left[\mathfrak{p}_{k+1}(\mathfrak{v}), \sum_{\mathfrak{w} \in \mathcal{J}} \mathfrak{p}_{l}(\mathfrak{w})\right] } & =\left[\left[\mathfrak{p}_{k}(\tilde{\mathfrak{v}}), v_{k+1}\right], \sum_{\mathfrak{w} \in \mathcal{J}} \mathfrak{p}_{l}(\mathfrak{w})\right] \\
& =-\left[\left[v_{k+1}, \sum_{\mathfrak{w} \in \mathcal{J}} \mathfrak{p}_{l}(\mathfrak{w})\right], \mathfrak{p}_{k}(\tilde{\mathfrak{v}})\right]-\left[\left[\sum_{\mathfrak{w} \in \mathcal{J}} \mathfrak{p}_{l}(\mathfrak{w}), \mathfrak{p}_{k}(\tilde{\mathfrak{v}})\right], v_{k+1}\right] \\
& =-\left[\mathfrak{p}_{k}(\tilde{\mathfrak{v}}), \sum_{\mathfrak{w} \in \mathcal{J}} \mathfrak{p}_{l+1}\left(\left(\mathfrak{w}, v_{k+1}\right)\right)\right]+\sum_{\mathfrak{w} \in \mathcal{J}}\left[\left[\mathfrak{p}_{k}(\tilde{\mathfrak{v}}), \mathfrak{p}_{l}(\mathfrak{w})\right], v_{k+1}\right] .
\end{aligned}
$$

All addends of the previous sum belong to $\mathbf{Y}_{k+l+1}$ by the inductive assumption, hence establishing conditions (5.1). The linear subspace

$$
V=\mathbf{Y}_{1}+\mathbf{Y}_{2}+\cdots+\mathbf{Y}_{v} \subset \mathbb{M}
$$


is closed under dilations, since so are all the subspaces $\mathbf{Y}_{j}$. Conditions (5.1) immediately show that $V$ is a Lie subalgebra of $\mathbb{M}$. It turns out that its closure

$$
\mathbb{V}=\bar{V}
$$

is a Banach Lie subalgebra, which is also closed under dilations, due to the continuity of the Lie product and of dilations. Then, the same argument in the proof of [26, Proposition 7.2] shows that $\mathbb{V}$ is actually a direct sum of subspaces $U_{1}, \ldots, U_{v}$ with $\left[U_{i}, U_{j}\right] \subset U_{i+j}$ and $U_{i+j}=\{0\}$ whenever $i+j>v$. Since the group operation is given by the Baker-CampbellHausdorff formula, it turns out that $\mathbb{V}$ is a Banach homogeneous subgroup of $\mathbb{M}$.

To show that $\mathbb{V}$ is also separable, we first consider the countable subsets

$$
\langle\langle\mathcal{N}\rangle\rangle_{k}=\left\{\sum_{v \in \mathcal{I}} \mathfrak{p}_{k}(v): \mathcal{I} \subset(\langle\langle\mathcal{N}\rangle\rangle)^{k} \text { is finite }\right\}
$$

where $2 \leq k \leq v$ and define the closed subset

$$
\mathbb{W}=\overline{\langle\langle\mathcal{N}\rangle\rangle+\langle\langle\mathcal{N}\rangle\rangle_{2}+\cdots+\langle\langle\mathcal{N}\rangle\rangle_{v}} .
$$

Clearly $\mathbb{W}$ is separable, being all the addends $\langle\langle\mathcal{N}\rangle\rangle$ and $\langle\langle\mathcal{N}\rangle\rangle_{k}$ countable. The simple inclusion $\langle\langle\mathcal{N}\rangle\rangle_{k} \subset \mathbf{Y}_{k}$ for $k \geq 2$ joined with $\mathbf{Y}_{1}=\overline{\langle\langle\mathcal{N}\rangle\rangle}$ immediately lead us to the following:

$$
\langle\langle\mathcal{N}\rangle\rangle+\langle\langle\mathcal{N}\rangle\rangle_{2}+\cdots+\left\langle\left\langle\mathcal{N}_{\nu}\right\rangle\right\rangle_{v} \subset V
$$

that gives $\mathbb{W} \subset \mathbb{V}$.

The opposite inclusion follows from the following:

$$
\mathbb{W} \supset V \text {. }
$$

To prove this, we consider any element

$$
w=v+\sum_{k=2}^{v} \sum_{\mathfrak{v} \in \mathcal{I}_{k}} \mathfrak{p}_{k}(\mathfrak{v}) \in V
$$

with $v \in \mathbf{Y}_{1}$ and $\mathcal{I}_{k}$ finite subset of $\mathbf{Y}_{1}^{k}$. Since $\overline{\langle\langle\mathcal{N}\rangle\rangle}=\mathbf{Y}_{1}$, we find a sequence $\left\{y_{n}\right\}$ of $\langle\langle\mathcal{N}\rangle\rangle$ with $y_{n} \rightarrow v$. For $k \geq 2$ and any $\mathfrak{v} \in \mathcal{I}_{k}$, we find a sequence $\left\{\mathfrak{v}_{n}\right\} \subset(\langle\langle\mathcal{N}\rangle\rangle)^{k}$ such that $\mathfrak{v}_{n} \rightarrow \mathfrak{v}$. By the continuity of $\mathfrak{p}$, we get

$$
\mathfrak{p}_{k}\left(\mathfrak{v}_{n}\right) \rightarrow \mathfrak{p}_{k}(\mathfrak{v}) \quad \text { as } n \rightarrow \infty .
$$

We conclude that

$$
z_{n}=y_{n}+\sum_{k=2}^{v} \sum_{\mathfrak{v} \in \mathcal{I}_{k}} \mathfrak{p}_{k}\left(\mathfrak{v}_{n}\right) \in\langle\langle\mathcal{N}\rangle\rangle+\langle\langle\mathcal{N}\rangle\rangle_{2}+\cdots+\langle\langle\mathcal{N}\rangle\rangle_{\mathcal{V}}
$$

and $z_{n} \rightarrow w \in \mathbb{W}$, showing the validity of (5.2) and then concluding the proof.

Theorem 5.2 If $A \subset \mathbb{G}$ and $f: A \rightarrow \mathbb{M}$ is continuous, then there exists a separable Banach homogeneous group $\mathbb{M}_{0} \subset \mathbb{M}$ such that $f(A) \subset \mathbb{M}_{0}$.

Proof Let $\mathcal{N} \subset \mathbb{M}$ be a countable subset such that $f(A) \subset \overline{\mathcal{N}}$. Then, Theorem 5.1 provides us with a separable Banach homogeneous group $\mathbb{M}_{0} \subset \mathbb{M}$ containing $\overline{\mathcal{N}}$, therefore concluding the proof. 


\subsection{Proof of Lemma 3.1}

Fix $b>0$. Since each map $\pi_{i}$ is continuous and linear, the following inequality holds for $|x| \leq b$, where $c>0$ only depends on $\mathbb{M}$ :

$$
\begin{aligned}
\left|\pi_{i}(x)\right|^{1 / i} \leq c^{1 / i}|x|^{1 / i} & =c^{1 / i}(1+b)^{1 / i}\left(\frac{|x|}{1+b}\right)^{1 / i} \\
& \leq c^{1 / i}(1+b)^{\frac{1}{i}-\frac{1}{v}}|x|^{1 / v} \\
& \leq \max \{c, 1\}(1+b)^{1-\frac{1}{v}}|x|^{1 / v},
\end{aligned}
$$

By (2.3) and (5.3), there exists $C_{1, b}>0$, depending on $b$, such that

$$
\|x\| \leq C_{1, b}|x|^{1 / \nu} \text { for }|x| \leq b .
$$

Conversely, from (2.3) one may easily see that there exists $C_{2, b}>0$ such that

$$
|x| \leq C_{2, b} \text { for }\|x\| \leq b .
$$

The same formula also yields

$$
|x| \leq \sum_{i=1}^{v}\left|\pi_{i}(x)\right| \leq \max _{1 \leq i \leq v}\left\{\frac{1}{\left(\sigma_{i}\right)^{i}}\right\} \sum_{i=1}^{v}\left(C_{1, b} b^{1 / v}\right)^{i-1}\|x\|=C_{3, b}\|x\| \quad \text { for }|x| \leq b .
$$

We observe that for the addends in (2.2) to be non-zero, the iterated nonassociative product must always start from the factor $x \circ y$ or $y \circ x$. Thus, the continuity of the Lie bracket yields

$$
|\underbrace{x \circ \cdots \circ x}_{p_{1} \text { times }} \circ \overbrace{y \circ \cdots \circ y}^{q_{1} \text { times }} \circ \cdots \circ \underbrace{x \circ \cdots \circ x}_{p_{k} \text { times }} \circ \overbrace{y \circ \cdots \circ y}^{q_{k} \text { times }}| \leq C^{m-2} b^{m-2}|[x, y]|
$$

where $\sum_{i=1}^{k}\left(p_{i}+q_{i}\right)=m$, and we have assumed in addition that $|x|,|y| \leq b$ for some $b>0$.

Claim 1 Let $b>0$. Then, there exists $C_{4, b}>0$, depending on $b$, such that

$$
\left\|y^{-1} x y\right\| \leq C_{4, b}|x|^{1 / v} \quad \text { for }|x|,|y| \leq b .
$$

Proof Recalling $y^{-1}=-y$ and applying Dynkin's formula with the pairs $-y, x y$ then $x, y$, we obtain

$$
y^{-1} x y=x+\sum_{m=2}^{v} P_{m}(x, y)+\sum_{m=2}^{v} P_{m}(-y, x y) .
$$

Taking into account (5.7), it is easy to see that there exists $C_{5, b}>0$, such that

$$
\left|y^{-1} x y\right| \leq C_{5, b}|x|+\left|\sum_{m=2}^{v} P_{m}(-y, x y)\right| .
$$

Another application of (5.7), joined with (2.2), gives a constant $C_{6, b}>0$ such that

$$
|x y| \leq C_{6, b} \quad \text { for }|x|,|y| \leq b .
$$

Again using (5.7), it follows that

$$
\left|P_{m}(-y, x y)\right| \leq C_{7, b}|[y, x y]|
$$


for each $m=2, \ldots, v$ and a suitable constant $C_{7, b}>0$, depending on $b$. Thus, we have proved the existence of $C_{8, b}>0$, depending on $b$, such that

$$
\left|y^{-1} x y\right| \leq C_{8, b}(|x|+|[y, x y]|) .
$$

Now, we observe that

$$
[y, x y]=\left[y, x+\sum_{m=2}^{v} P_{m}(x, y)\right]=[y, x]+\left[y, \sum_{m=2}^{v} P_{m}(x, y)\right] .
$$

This together with the continuity of $[\cdot, \cdot]$ leads us to the estimate

$$
|[y, x y]| \leq C_{0}\left(|x||y|+|y| \sum_{m=2}^{v}\left|P_{m}(x, y)\right|\right)
$$

where $C_{0}>0$ only depends on the Banach norm chosen on $\mathbb{M}$. As a consequence, there exists $C_{9, b}>0$ such that

$$
|[y, x y]| \leq C_{9, b}|x| \text { for }|x|,|y| \leq b .
$$

The previous inequality joint with (5.11) gives $\left|y^{-1} x y\right| \leq C_{10, b}$ for some $C_{10, b}>0$ depending on $b$. This bound on $\left|y^{-1} x y\right|$ allows us to apply (5.4), and that joined with (5.11) and (5.12) gives (5.8).

Claim 2 Let $N$ be a positive integer and let $A_{j}, B_{j} \in \mathbb{M}$ with $j=1, \ldots, N$. Let $b>0$ be such that $\left|B_{j} B_{j+1} \cdots B_{N}\right| \leq b$ and $\left|B_{j}^{-1} A_{j}\right| \leq b$ for every $j=1, \ldots, N$. Then, there exists $C>0$ such that

$$
\rho\left(A_{1} A_{2} \cdots A_{N}, B_{1} B_{2} \cdots B_{N}\right) \leq \rho\left(A_{N}, B_{N}\right)+C \sum_{j=1}^{N-1}\left|A_{j}^{-1} B_{j}\right|^{1 / \nu} .
$$

Proof. Define $\hat{B}_{j}=B_{j} B_{j+1} \cdots B_{N}$ and $\hat{A}_{j}=A_{j} A_{j+1} \cdots A_{N}$. Using the left invariance of $\rho$ and Claim 1 , we obtain

$$
\begin{aligned}
\rho\left(\hat{A}_{1}, \hat{B}_{1}\right)=\rho\left(\hat{A}_{2}, A_{1}^{-1} B_{1} \hat{B}_{2}\right) & \leq \rho\left(\hat{A}_{2}, \hat{B}_{2}\right)+\left\|\hat{B}_{2}^{-1} A_{1}^{-1} B_{1} \hat{B}_{2}\right\| \\
& \leq \rho\left(\hat{A}_{2}, \hat{B}_{2}\right)+C_{4, b}\left|A_{1}^{-1} B_{1}\right|^{1 / v} \\
& \leq \rho\left(\hat{A}_{3}, \hat{B}_{3}\right)+C_{4, b}\left|A_{2}^{-1} B_{2}\right|^{1 / v}+C_{4, b}\left|A_{1}^{-1} B_{1}\right|^{1 / v} \\
& \leq \rho\left(A_{N}, B_{N}\right)+C \sum_{j=1}^{N-1}\left|A_{j}^{-1} B_{j}\right|^{1 / v} .
\end{aligned}
$$

From the hypotheses of Lemma 3.1, taking into account (5.5), we see

$$
\left|B_{j} B_{j+1} \cdots B_{N}\right| \leq C_{2, b} \text { and }\left|A_{j}^{-1} B_{j}\right| \leq C_{2, b} \quad \text { for } j=1, \ldots, N .
$$

Hence, we may apply Claim 2. Recalling that $\rho\left(A_{N}, B_{N}\right) \leq b$ and using (5.6), we obtain

$$
\begin{aligned}
\rho\left(A_{1} A_{2} \cdots A_{N}, B_{1} B_{2} \cdots B_{N}\right) & \leq \rho\left(A_{N}, B_{N}\right)+C_{3, C_{2, b}} \sum_{j=1}^{N-1}\left\|A_{j}^{-1} B_{j}\right\|^{1 / v} \\
& \leq C_{b} \sum_{j=1}^{N} \rho\left(A_{j}, B_{j}\right)^{1 / v}
\end{aligned}
$$

where $C_{b}=\max \left\{b^{1-\frac{1}{v}}, C_{3, C_{2, b}}\right\}$. This concludes the proof of Lemma 3.1. 


\subsection{Measurability of points where a directional derivative exists}

The aim of this section is to prove that the set $D_{f, \zeta}$ in the proof of Theorem 2.16 is measurable. Recall that $f: A \rightarrow \mathbb{M}$ is Lipschitz and $A \subset \mathbb{G}$ is measurable. It was shown earlier that the set $A_{\zeta}$ of points at which $A$ is dense in direction $\zeta$ is measurable and $\mu\left(A \backslash A_{\zeta}\right)=0$. The set $D_{f, \zeta}$ is the subset of points in $A_{\zeta}$ where $f$ is differentiable in direction $\zeta$. By Theorem 5.2, we can assume that the target $\mathbb{M}$ is a separable Banach homogeneous group. Fix $z_{i} \in \mathbb{M}$ with $\overline{\left\{z_{i}: i \in \mathbb{N}\right\}}=\mathbb{M}$. Let $P_{i}(z)=\rho\left(z, z_{i}\right)$ for $z \in \mathbb{M}$. For $y \in A$ and $t>0$, define measurable functions $g_{t, y,+}^{i}, g_{t, y,-}^{i}: A_{\zeta} \rightarrow \mathbb{R} \cup\{+\infty,-\infty\}$ by:

$$
g_{t, y, \pm}^{i}(x)= \begin{cases}P_{i}\left(\delta_{1 / t}\left(f(x)^{-1} f(y)\right)\right) & \text { if } y \in A \cap B\left(x \delta_{t} \zeta, t^{2}\right) \\ \pm \infty & \text { if } y \in A \backslash B\left(x \delta_{t} \zeta, t^{2}\right) .\end{cases}
$$

Fix a countable set $\mathcal{S} \subset A$ with $A \subset \overline{\mathcal{S}}$. Define

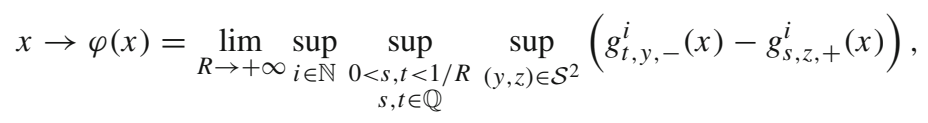

which is well defined and measurable on $A_{\zeta}$. We claim that $f$ is differentiable at $x$ in direction $\zeta$ if and only if $\varphi(x)=0$.

First, suppose $x \in A_{\zeta}$ and $f$ is differentiable at $x$ in direction $\zeta$. Proposition 2.14 implies that

$$
\lim _{t \downarrow 0, x \delta_{t} \zeta \in A} \delta_{1 / t}\left(f(x)^{-1} f\left(x \delta_{t} \zeta\right)\right)=\partial^{+} f(x, \zeta) .
$$

Since $x$ is a density point of $A$ in direction $\zeta$, there exists $T_{t}>0$ with $x \delta_{T_{t}} \zeta \in A$ and $T_{t} / t \rightarrow 1$ as $t \downarrow 0$. For $y \in B\left(x \delta_{t} \zeta, t^{2}\right)$, let

$$
E_{x, y, t}=\delta_{1 / t}\left(f\left(x \delta_{T_{t}} \zeta\right)^{-1} f(y)\right)
$$

Taking into account that $\zeta$ is horizontal, we have

$$
\rho\left(E_{x, y, t}\right) \leq L \frac{d\left(x \delta_{T_{t}} \zeta, y\right)}{t}<\frac{L}{t}\left(\left|T_{t}-t\right| d(\zeta)+t^{2}\right)=L \theta_{t},
$$

where $\theta_{t} \rightarrow 0$ as $t \downarrow 0$. We set

$$
(\delta f)_{x, \zeta, t}=\delta_{1 / t}\left(f(x)^{-1} f\left(x \delta_{T_{t}} \zeta\right)\right)
$$

observing that $(\delta f)_{x, \zeta, t} \rightarrow \partial^{+} f(x, \zeta)$ as $t \downarrow 0$. The following estimates hold

$$
\begin{aligned}
& \left|P_{i}\left(\delta_{1 / t}\left(f(x)^{-1} f(y)\right)\right)-P_{i}\left((\delta f)_{x, \zeta, t}\right)\right| \\
& \quad=\left|P_{i}\left((\delta f)_{x, \zeta, t} E_{x, y, t}\right)-P_{i}\left((\delta f)_{x, \zeta, t}\right)\right| \leq \rho\left(E_{x, y, t}\right)<L \theta_{t}
\end{aligned}
$$

uniformly in $y \in B\left(x \delta_{t} \zeta, t^{2}\right)$ and $i \in \mathbb{N}$. Since $P: \mathbb{M} \rightarrow \ell^{\infty}, z \rightarrow\left(P_{i}(z)\right)_{i \in \mathbb{N}}$ is an isometric embedding, we get

$$
\sup _{i \in \mathbb{N}}\left|P_{i}\left((\delta f)_{x, \zeta, t}\right)-P_{i}\left(\partial^{+} f(x, \zeta)\right)\right| \rightarrow 0 \quad \text { as } t \downarrow 0 .
$$

If we fix $\varepsilon>0$, then for some $R_{\varepsilon}>0$ we obtain

$$
\left|P_{i}\left(\delta_{1 / t}\left(f(x)^{-1} f(y)\right)\right)-P_{i}\left(\partial^{+} f(x, \zeta)\right)\right|<\varepsilon / 2
$$


for $0<t<1 / R_{\varepsilon}, i \in \mathbb{N}$ and $y \in B\left(x \delta_{t} \zeta, t^{2}\right)$. In particular, for $y \in B\left(x \delta_{t} \zeta, t^{2}\right)$ and $z \in B\left(x \delta_{s} \zeta, s^{2}\right)$, it follows that

$$
\left|P_{i}\left(\delta_{1 / t}\left(f(x)^{-1} f(y)\right)\right)-P_{i}\left(\delta_{1 / s}\left(f(x)^{-1} f(z)\right)\right)\right|<\varepsilon
$$

for $0<t, s<1 / R_{\varepsilon}$ and $i \in \mathbb{N}$. The fact that $A$ is dense at $x$ in direction $\zeta$ implies that, up to taking a larger $R_{\mathcal{E}}$, the following

$$
\sup _{\substack{0<s, t<1 / R_{\varepsilon} \\ s, t \in \mathbb{Q}}} \sup _{(y, z) \in \mathcal{S}^{2}}\left(g_{t, y,-}^{i}(x)-g_{s, z,+}^{i}(x)\right)
$$

exactly equals

$$
\sup _{\substack{0<s, t<1 / R_{\varepsilon} \\ s, t \in \mathbb{Q}}} \sup _{\substack{(y, z) \in \mathcal{S}^{2} \\ y \in B\left(x \delta_{t} \zeta, t^{2}\right), z \in B\left(x \delta_{s} \zeta, s^{2}\right)}} P_{i}\left(\delta_{1 / t}\left(f(x)^{-1} f(y)\right)\right)-P_{i}\left(\delta_{1 / s}\left(f(x)^{-1} f(z)\right)\right)<\varepsilon
$$

for every $i \in \mathbb{N}$. This proves that $\varphi(x)=0$.

Conversely, we assume that $\varphi(x)=0$ and wish to prove that $f$ is differentiable at $x$ in direction $\zeta$. Let $\varepsilon>0$ be arbitrarily fixed, and choose $R_{\varepsilon}>0$ such that

$$
\sup _{i \in \mathbb{N}} \sup _{\substack{0<s, t<1 / R_{\varepsilon} \\ s, t \in \mathbb{Q}}} \sup _{(y, z) \in \mathcal{S}^{2}}\left(g_{t, y,-}^{i}(x)-g_{s, z,+}^{i}(x)\right)<\varepsilon .
$$

Observing that in the expression (5.14) we can exchange $(t, y)$ with $(s, z)$, if $y \in B\left(x \delta_{t} \zeta, t^{2}\right)$ and $z \in B\left(x \delta_{s} \zeta, s^{2}\right)$ we get

$$
\left|P_{i}\left(\delta_{1 / t}\left(f(x)^{-1} f(y)\right)\right)-P_{i}\left(\delta_{1 / s}\left(f(x)^{-1} f(z)\right)\right)\right| \leq \varepsilon
$$

for $0<s, t \leq 1 / R_{\varepsilon}$. Since $x \in A$ is a density point in direction $\zeta$, we may consider again $T_{t}>0$ such that $x \delta_{T_{t}} \zeta \in A$ and $T_{t} / t \rightarrow 1$ as $t \downarrow 0$. Fix $\delta_{0}>0$ such that $T_{t}<2 t$ for $0<t \leq \delta_{0}$. Whenever $0<s, t<1 / 2 R_{\varepsilon}$, we have $0<T_{s}, T_{t}<1 / R_{\varepsilon}$ and then

$$
\left|P_{i}\left(\delta_{1 / T_{t}}\left(f(x)^{-1} f\left(x \delta_{T_{t}} \zeta\right)\right)\right)-P_{i}\left(\delta_{1 / T_{s}}\left(f(x)^{-1} f\left(x \delta_{T_{s}} \zeta\right)\right)\right)\right| \leq \varepsilon
$$

for all $i \in \mathbb{N}$. In particular, there exists

$$
l_{i}=\lim _{t \downarrow 0} P_{i}\left(\delta_{1 / T_{t}}\left(f(x)^{-1} f\left(x \delta_{T_{t}} \zeta\right)\right)\right),
$$

and we can pass to the limit in (5.15) with respect to $s \downarrow 0$ that yields

$$
\left|P_{i}\left(\delta_{1 / T_{t}}\left(f(x)^{-1} f\left(x \delta_{T_{t}} \zeta\right)\right)\right)-l_{i}\right| \leq \varepsilon .
$$

In particular, $w=\left(l_{i}\right) \in \ell^{\infty}$ and

$$
\left\|P\left(\delta_{1 / T_{t}}\left(f(x)^{-1} f\left(x \delta_{T_{t}} \zeta\right)\right)\right)-w\right\|_{\ell^{\infty}} \rightarrow 0 \quad \text { as } t \downarrow 0 .
$$

Hence, the following limit exists

$$
\lim _{t \downarrow 0} \delta_{1 / T_{t}}\left(f(x)^{-1} f\left(x \delta_{T_{t}} \zeta\right)\right) .
$$

By Remark 2.11, this shows that $f$ is differentiable at $x$ in direction $\zeta$.

We conclude that $D_{f, \zeta}=\left\{x \in A_{\zeta}: \varphi(x)=0\right\}$, from which the measurability of $\varphi$ gives our claim. 


\section{References}

1. Agrachev, A., Barilari, D., Boscain, U.: A comprehensive introduction to sub-riemannian geometry. In: Cambridge Studies in Advanced Mathematics. Cambridge University Press (2019)

2. Alberti, G., Marchese, A.: On the differentiability of Lipschitz functions with respect to measures in the Euclidean space. Geom. Funct. Anal. 26(1), 1-66 (2016)

3. Ambrosio, L., Kirchheim, B.: Rectifiable sets in metric and Banach spaces. Math. Ann. 318(3), 527-555 (2000)

4. Bate, D.: Structure of measures in Lipschitz differentiability spaces. J. Am. Math. Soc. 28(2), 421-482 (2015)

5. Bellaiche, A.: The tangent space in sub-Riemannian geometry. J. Math. Sci. 83(4), 461-476 (1994)

6. Bonfiglioli, A., Lanconelli, E., Uguzzoni, F.: Stratified Lie Groups and Potential Theory for Their Sublaplacians. Springer Monographs in Mathematics 26. Springer, New York (2007)

7. Cheeger, J.: Differentiability of Lipschitz functions on metric measure spaces. Geom. Funct. Anal. 9(3), 428-517 (1999)

8. Cheeger, J., Kleiner, B.: Differentiability of Lipschitz maps from metric measure spaces to Banach spaces with the Radon-Nikodym property. Geom. Funct. Anal. 19(4), 1017-1028 (2009)

9. Citti, G., Manfredini, M., Pinamonti, A., Serra Cassano, F.: Smooth approximation for intrinsic Lipschitz functions in the Heisenberg group. Calc. Var. Partial Differ. Equ. 49(3-4), 1279-1308 (2014)

10. Citti, G., Manfredini, M., Pinamonti, A., Serra, Cassano, F.: Poincaré-type inequality for Lipschitz continuous vector fields. J. Math. Pures Appl. (9) 105(3), 265-292 (2016)

11. Capogna, L., Danielli, D., Pauls, S., Tyson, J.: An Introduction to the Heisenberg Group and the SubRiemannian Isoperimetric Problem. In: Progress in Mathematics, vol. 259. Birkhäuser Verlag, Basel (2007)

12. Federer, H.: Geometric Measure Theory, Die Grundlehren der mathematischen Wissenschaften. Springer, New York (1969)

13. Folland, G.B., Stein, E.: Hardy Spaces on Homogeneous Groups. Princeton University Press, Princeton (1982)

14. Folland, G.B.: Subelliptic estimates and function spaces on nilpotent Lie groups. Ark. Mat. 13(1-2), 161-207 (1975)

15. Franchi, B., Serapioni, R.: Intrinsic Lipschitz graphs within Carnot groups. J. Geom. Anal. 26(3), 19461994 (2016)

16. Franchi, B., Serapioni, R., Serra Cassano, F.: Differentiability of intrinsic Lipschitz functions within Heisenberg groups. J. Geom. Anal. 21(4), 1044-1084 (2011)

17. Gromov, M.: Carnot-Carathéodory spaces seen from within. Prog. Math. 144, 79-323 (1996)

18. Hajlasz, P., Malekzadeh, S.: On conditions for unrectifiability of a metric space. Anal. Geom. Metr. Spaces 3(1), 1-14 (2015)

19. Heinonen, J.: Lectures on Analysis on Metric Spaces. Universitext. Springer, New York (2001)

20. Le Donne, E., Pinamonti, A., Speight, G.: Universal differentiability sets and maximal directional derivatives in Carnot groups. J. Math. Pures Appl. 121, 83-112 (2019)

21. Le Donne, E., Li, S., Moisala, T.: Gateaux differentiability on infinite-dimensional Carnot groups. arXiv: 1812.07375

22. Lindenstrauss, J., Preiss, D.: On Fréchet differentiability of Lipschitz maps between Banach spaces. Ann. Math. 157, 257-288 (2003)

23. Lindenstrauss, J., Preiss, D., Tiser, J.: Fréchet Differentiability of Lipschitz Functions and Porous Sets in Banach Spaces. Annals of Mathematics Studies 179. Princeton University Press, Princeton (2012)

24. Magnani, V.: Differentiability and area formula on stratified Lie groups. Houst. J. Math. 27(2), 297-323 (2001)

25. Magnani, V.: Unrectifiability and rigidity in stratified groups. Arch. Math. 83(6), 568-576 (2004)

26. Magnani, V.: Towards differential calculus in stratified groups. J. Aust. Math. Soc. 95(1), 76-128 (2013)

27. Magnani, V., Rajala, T.: Radon-Nikodym property and area formula for Banach homogeneous group targets. Int. Math. Res. Not. IMRN 2014(23), 6399-6430 (2014). https://academic.oup.com/imrn/article/ 2014/23/6399/747805/

28. Montgomery, R.: A tour of subriemannian geometries, their geodesics and applications. In: Mathematical Surveys and Monographs, vol. 91. American Mathematical Society, Providence, RI (2002)

29. Pansu, P.: Metriques de Carnot-Carathéodory et quasiisometries des espaces symetriques de rang un. Ann. Math. 129(1), 1-60 (1989)

30. Pinamonti, A., Speight, G.: A measure zero universal differentiability set in the Heisenberg group. Math. Ann. 368(1), 233-278 (2017) 
31. Pinamonti, A., Speight, G.: Porosity, differentiability and Pansu's theorem. J. Geom. Anal. 27(3), 20552080 (2017)

32. Pinamonti, A., Speight, G.: Structure of porous sets in Carnot groups. Ill. J. Math. 61(1-2), 127-150 (2017)

33. Pinamonti, A., Speight, G.: A measure zero UDS in the Heisenberg group. Bruno Pini Math. Anal. Semin. 7, 85-96 (2016)

34. Preiss, D.: Differentiability of Lipschitz functions on Banach spaces. J. Funct. Anal. 91(2), 312-345 (1990)

35. Preiss, D., Speight, G.: Differentiability of Lipschitz functions in Lebesgue null sets. Invent. Math. 199(2), 517-559 (2015)

36. Preiss, D., Zajicek, L.: Directional derivatives of Lipschitz functions. Isr. J. Math. 125, 1-27 (2001)

37. Semmes, S.: On the nonexistence of bi-Lipschitz parameterizations and geometric problems about $A_{\infty^{-}}$ weights. Rev. Mat. Iberoam. 12(2), 337-410 (1996)

38. Serra Cassano, F.: Some topics of geometric measure theory in Carnot groups. In: Geometry, analysis and dynamics on sub-Riemannian manifolds, vol. 1. EMS Ser. Lect. Math. Eur. Math. Soc., Zürich, pp. $1-121$ (2016)

39. Zajicek, L.: Porosity and $\sigma$-porosity. Real Anal. Exch. 13(2), 314-350 (1987/1988)

40. Zajicek, L.: On $\sigma$-porous sets in abstract spaces. Abstr. Appl. Anal. 5, 509-534 (2005)

Publisher's Note Springer Nature remains neutral with regard to jurisdictional claims in published maps and institutional affiliations. 